\title{
The r-Process of Nucleosynthesis: The Puzzle Is Still with Us
}

\author{
Marcel Arnould and Stephane Goriely \\ Université Libre de Bruxelles \\ Belgium
}

\section{Introduction}

Through the increasing number and quality of astronomical observations, our picture of the composition of the various constituents of the Universe is getting quickly more and more complete, and concomitantly more and more complex. Despite this spectacular progress, the solar system (hereafter SoS) continues to provide a body of abundance data whose quantity, quality and coherence remain unmatched. This concerns especially the heavy elements (defined here as those with atomic numbers in excess of the value $Z=26$ corresponding to iron), and in particular their isotopic compositions, which are the prime fingerprints of astrophysical nuclear processes. Except in a few cases, these isotopic patterns indeed remain out of reach even of the most-advanced stellar spectroscopic techniques available today. No wonder then that, from the early days of its development, the theory of nucleosynthesis has been deeply rooted in the SoS composition, especially in the heavy element domain.

Since the early days of the development of the theory of nucleosynthesis, it has been proved operationally rewarding to introduce three categories of heavy nuclides referred to as s-, $\mathrm{p}-$, and r-nuclides. This splitting corresponds to the 'topology' of the chart of the nuclides, which exhibits three categories of stable heavy nuclides: those located at the bottom of the valley of nuclear stability, called the s-nuclides, and those situated on the neutron-deficient or neutron-rich side of the valley, named the p- or r-nuclides, respectively. Three different mechanisms are called for to account for the production of these three types of stable nuclides. They are naturally referred to as the s-, r-, and p-processes. An extensive survey of the p-process can be found in Arnould \& Goriely (2003), while some aspects of the s-process are the subject of another chapter of this book.

The main aim of this chapter is to provide a rather brief summary of some selected astrophysics and nuclear physics aspects of the r-process already reviewed in detail by Arnould et al. (2007), but that we consider to be worth emphasizing again for one reason or another. Some update of this review is also proposed, based on papers published after September 2006.

\section{The bulk solar system abundances of the r-nuclides: too often neglected severe intricacies}

The bulk SoS material has long been recognized to be made of a well-mixed blend of many nucleosynthesis contributions over the approximate $10 \mathrm{~Gy}$ that have elapsed between the formations of the Galaxy and of the SoS. Since the nineteen fifties, much effort has been 
devoted to the highly complex derivation of a meaningful set of elemental abundances representative of this material at the SoS formation some $4.6 \mathrm{~Gy}$ ago. This research has led to refinements over the years, but not to drastic changes (see (Lodders, 2010) for the most recent compilation).

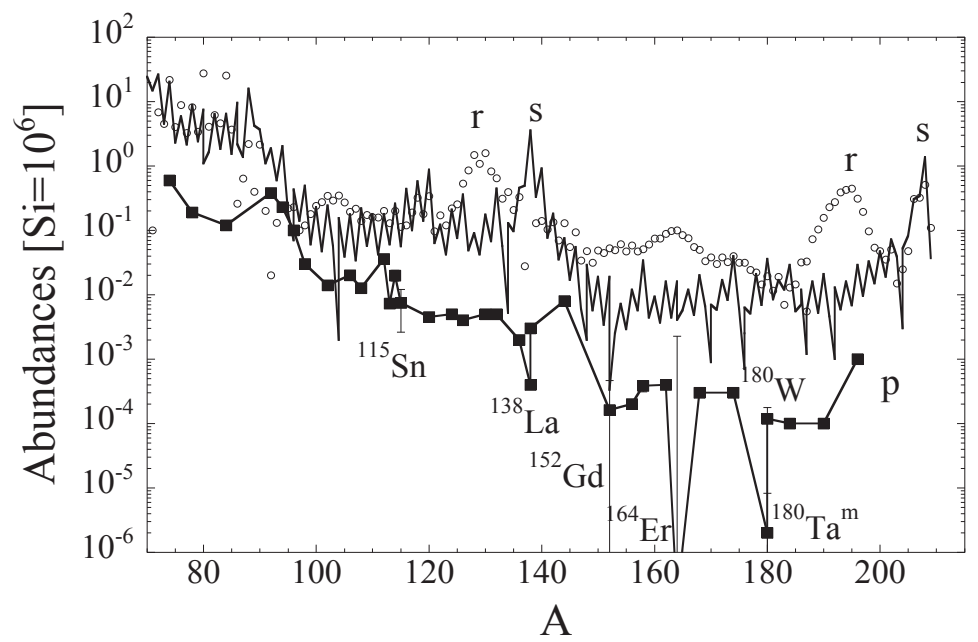

Fig. 1. Decomposition of the solar abundances of heavy nuclides into s-process (solid line), r-process (open circles) and p-process (squares) contributions. The uncertainties on the abundances of some p-nuclides due to a possible s-process contamination are represented by vertical bars (from (Arnould \& Goriely, 2003)). See Figs. 2 - 4 for the uncertainties in the sand r-nuclide data

As mentioned above, it is very useful to split the abundance distribution of the nuclides heavier than iron into three separate distributions giving the image of the SoS content of the p-, s- and r-nuclides. A rough representation of this splitting is displayed in Fig. 1. In its details, the procedure of decomposition is by far not as obvious as it might be thought from the very definition of the different types of the nuclides heavier than iron, and is to some extent dependent on the models for the synthesis of these nuclides. These models predict in particular that the stable nuclides located on the neutron-rich(deficient) side of the valley of nuclear stability are produced, to a first good approximation, by the $r-(p-)$ process only. These stable nuclides are naturally called ' $r$-only' and ' $p$-only' nuclides, and their abundances are deduced directly from the SoS abundances. The situation is much more intricate for the nuclides situated at the bottom of the valley of nuclear stability. Some of them are produced solely by the s-process, the typical flow of which is located very close to the valley (see the review of the s-process in this volume). They are referred to as 's-only' nuclides, and are encountered only when a stable $r$ - or p-isobar exists, which 'shields' the s-isobar from the $\mathrm{r}$ - and p-processes. As a result, only even- $Z$ heavy elements possess an s-only isotope. In the other cases, the nuclides at the bottom of the valley of nuclear stability may be produced by both the s- and the r-processes in proportions that vary from nuclide to nuclide. They are referred to as ' $\mathrm{sr}^{\prime}$ nuclides, and the relative contributions of the two processes to their abundances are the most intricate to evaluate ${ }^{1}$.

\footnotetext{
${ }^{1}$ The abundances of some nuclides may also result result from a contribution of the s- and p-processes, they are referred to as 'sp' nuclides
} 
Schematic SoS distributions of the p-, s- and r-nuclides are displayed in Fig. 1. It appears that about half of the heavy nuclei in the SoS material come from the s-process, and the other half from the r-process, whereas the p-process is responsible for the production of about 0.01 to 0.001 of the abundances of the s- and r-isobars. Some elements have their abundances dominated by an s- or r-nuclide. They are naturally referred to as s- or r-elements. Clearly, p-elements do not exist. These general characteristics are not drastically sensitive to the particular model used to obtain this splitting. In contrast, most details of the decomposition between the s- and r-contributions do, and many uncertainties remain. It is highly unfortunate that they are classically put under the rug, especially in view of the sometimes very detailed and far-reaching considerations that have the s-r SoS splitting as an essential starting point.

The canonical s-process model has been widely used to split the s- and r-contributions to the SoS material. In short, this model assumes that stellar material composed only of iron nuclei is subject to neutron densities and temperatures that remain constant over the whole period of the neutron irradiation. In addition, the SoS s-abundance pattern is viewed as originating from a superposition of two exponential distributions of the time-integrated neutron exposure $\tau_{\mathrm{n}}=\int_{0}^{t} N_{\mathrm{n}} v_{T} \mathrm{~d} t$ (where $v_{T}$ is the most probable relative neutron-nucleus velocity at temperature $T$, and $N_{n}$ is the neutron density). These distributions are traditionally held responsible for the so-called weak $(70 \lesssim A \lesssim 90)$ and main $(A \gtrsim 90)$ components of the s-process. A third exponential distribution is sometimes added in order to account for the $204<A \leq 209$ s-nuclides. Through an adequate fitting of the parameters of the two or three $\tau$-distributions, the superposition of the two or three resulting abundance components reproduces quite successfully the abundance distribution of the s-only nuclides in the SoS, from which it is concluded that the s-contribution to the sr-nuclides can be predicted reliably. It has to be stressed that this result is rooted only in the nuclear properties of the species involved in the s-process, and does not rely at all on specific astrophysics scenarios.

In spite of the claimed success of the canonical model, some of its basic assumptions deserve questioning. This concerns in particular a presumed exponential form for the distribution of the neutron exposures $\tau$, which has been introduced in view of its mathematical ease in abundance calculations. In addition, the canonical model makes it difficult to evaluate uncertainties of nuclear or observational nature. As a result, the concomitant uncertainties in the SoS r-abundances are traditionally not evaluated.

For about ten years, it has become fashionable to predict the s- and r-contributions from s-process calculations performed in the framework of some models for stars of various masses and initial compositions (e.g. Arlandini et al., 1999; Simmerer et al., 2004). We strongly suggest not to use this procedure for predicting the SoS s- and r-components. Large uncertainties indeed remain in the s-abundances predicted from all model stars. In addition, the SoS s-nuclide abundances result from a long evolution of the galactic composition that cannot be mimicked reliably enough.

A third approach has been proposed by Goriely (1997), and is referred to as the multi-event s-process model (MES). It cures to a large extent many of the shortcomings of the canonical model, and is in fact the only attempt so far to evaluate on a quantitative basis the uncertainties in the derived SoS s- and r-abundances. This makes quite incomprehensible the fact that it has remained largely unnoticed for more than ten years. In view of the importance to evaluate the uncertainties affecting the SoS distribution of the abundances of the r-nuclides, we review the MES in some detail. 
The MES relies on a superposition of a given number of canonical events, each of them being defined by a neutron irradiation on the ${ }^{56} \mathrm{Fe}$ seed nuclei during a time $t_{\text {irr }}$ at a constant temperature $T$ and a constant neutron density $N_{\mathrm{n}}$. In contrast to the canonical model, no hypothesis is made concerning any particular distribution of the neutron exposures. Only a set of canonical events that are considered as astrophysically plausible is selected a priori. We adopt here about 500 s-process canonical events covering ranges of astrophysical conditions that are identified as relevant by the canonical model, that is $1.5 \times 10^{8} \leq \mathrm{T} \leq 4 \times 10^{8} \mathrm{~K}$, $7.5 \leq \log N_{\mathrm{n}}\left[\mathrm{cm}^{-3}\right] \leq 10$, and 40 chosen $t_{\text {irr }}$-values, corresponding to evenly distributed values of $n_{\text {cap }}$ in the $5 \leq n_{\text {cap }} \leq 150$ range, where

$$
n_{\text {cap }}=\sum_{Z, A} A N_{Z, A}\left(t=t_{\text {irr }}\right)-\sum_{Z, A} A N_{Z, A}(t=0)
$$

is the number of neutrons captured per seed nucleus $\left({ }^{56} \mathrm{Fe}\right)$ on the timescale $t_{\text {irr }}$, the summation extending over all the nuclides involved in the s-process. For each of the selected canonical events, the abundances $N_{Z, A}$ are obtained by solving a reaction network including 640 nuclear species between $\mathrm{Cr}$ and Po. Based on these calculated abundances, an iterative inversion procedure described in Goriely (1997) allows to identify a combination of events from the considered set that provides the best fit to the solar abundances of a selected ensemble of nuclides. This set includes 35 nuclides comprising the s-only nuclides, complemented with ${ }^{86} \mathrm{Kr}$ and ${ }^{96} \mathrm{Zr}$ (largely produced by the s-process in the canonical model), ${ }^{152} \mathrm{Gd}$ and ${ }^{164} \mathrm{Er}$ (not produced in the p-process, but able to be produced in solar abundances in the s-process (Arnould \& Goriely, 2003)), and ${ }^{208} \mathrm{~Pb}$ (possibly produced by the strong s-process component in the canonical model).

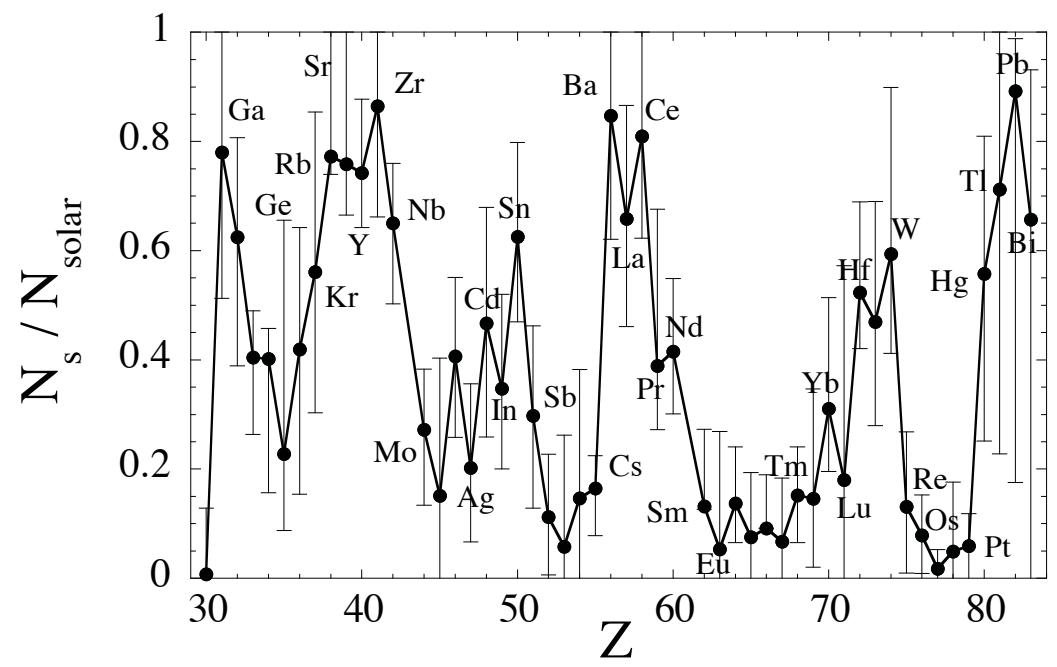

Fig. 2. The s-process contribution to the elemental SoS abundances $N_{\text {solar }}$ (Palme \& Beer, 1993) of the elements with $Z \geq 30$ predicted from the MES procedure. Uncertainties are represented by vertical bars

On grounds of the solar abundances of Palme \& Beer (1993), it is demonstrated in Goriely (1997) that the MES provides an excellent fit to the abundances of the 35 nuclides included 


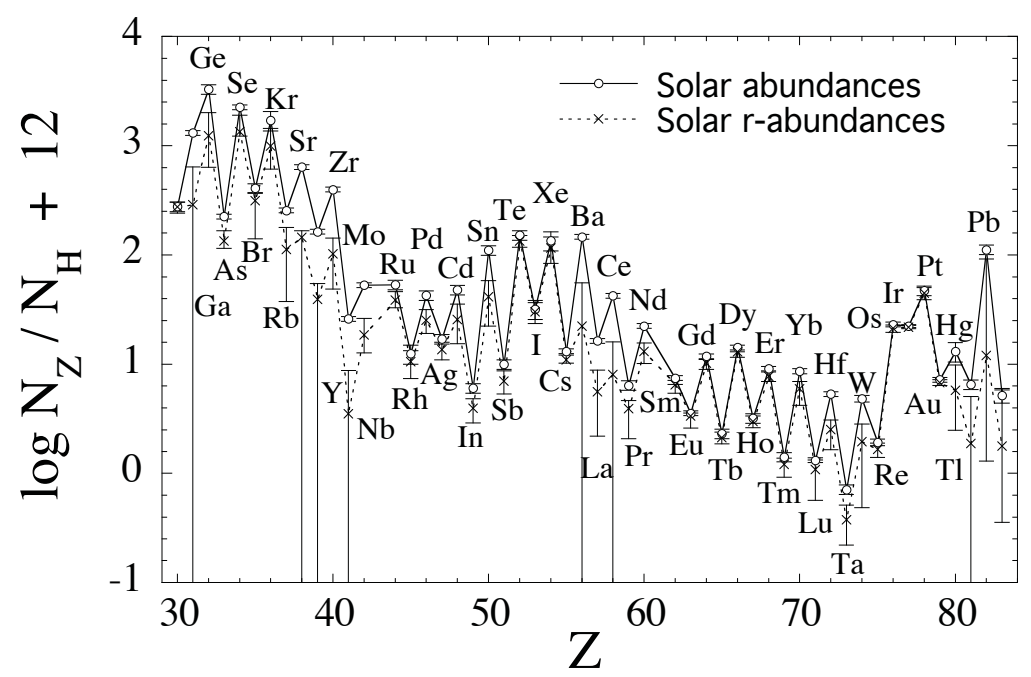

Fig. 3. SoS r-residuals and their uncertainties for the $Z \geq 30$ elements based on the s-abundances of Fig. 2. The abundances $N_{\mathrm{H}}$ and $N_{\mathrm{Z}}$ refer to hydrogen and to the element with atomic number $Z$

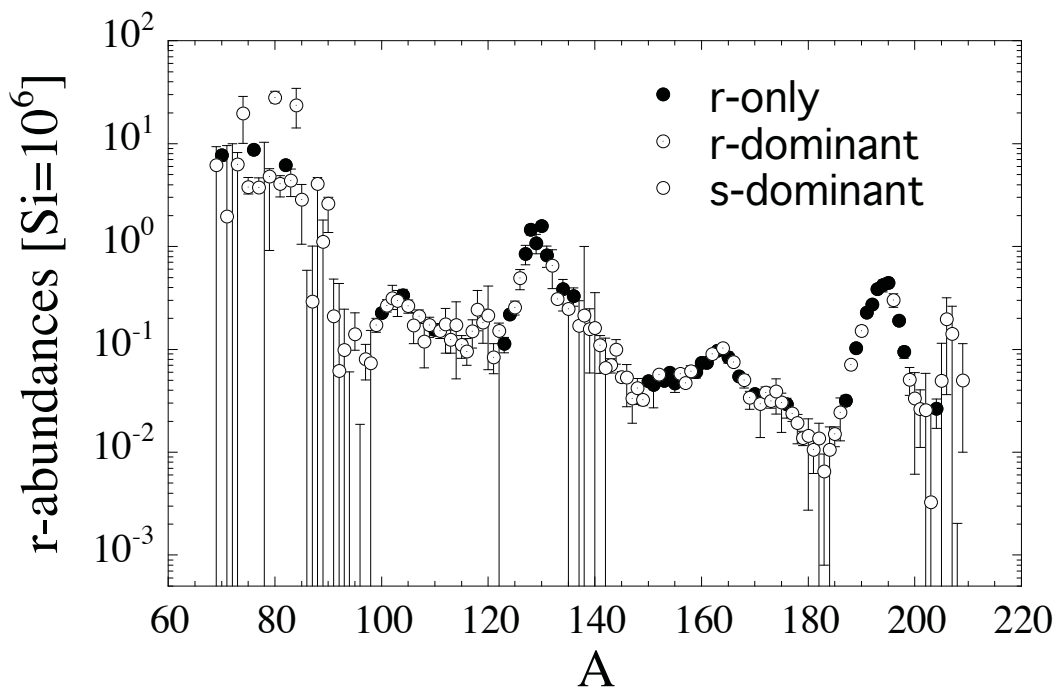

Fig. 4. SoS isotopic r-residuals corresponding to the elemental abundances of Fig. 3. Different symbols identify different relative levels of r-process contribution. The s-dominant nuclides are those predicted by MES to have more than $50 \%$ of their abundances produced by the s-process. The s-process contribution varies between 10 and $50 \%$ in the case of the r-dominant species, and does not exceed $10 \%$ for the r-only nuclides 
in the considered set of species, and in fact performs to a quite-similar overall quality as that of the exponential canonical model predictions of Palme \& Beer (1993) with a distribution of neutron irradiations that agrees qualitatively with the exponential distributions assumed in the canonical model, even though some deviations are noticed with respect to the canonical weak and strong components. ${ }^{2}$. An even better fit than in the canonical framework is obtained for the s-only nuclides (see Goriely, 1999, for details). The MES model is therefore expected to provide a decomposition of the solar abundances into their s-and r-components that is likely to be more reliable than the one derived from the canonical approach without any a priori assumption regarding the distribution of neutron exposures.

Compared with the canonical approach, the MES model has the additional major advantage of allowing a systematic study of the various uncertainties affecting the abundances derived from the parametric s-process model, and consequently the residual r-nuclide abundances. The uncertainties in these residuals have been studied in detail by Goriely (1999) from due consideration of the uncertainties in (i) the observed SoS abundances as given by Palme \& Beer (1993) (see footnote ${ }^{1}$ ), (ii) the experimental and theoretical radiative neutron-capture rates involved in the s-process network, and in (iii) the relevant $\beta$-decay and electron-capture rates. Total uncertainties resulting from a combination of (i) to (iii) have finally been evaluated. The r-residuals obtained from such a study for the elements with $Z \geq 30$ are displayed in Fig. 3 . Under the classical adoption of terrestrial isotopic compositions of the considered elements, the corresponding SoS isotopic r-residuals and their uncertainties have been calculated by Goriely (1999) and are shown in Fig. 4. They are presented in tabular form in Goriely (1999) and Arnould et al. (2007). Different situations can be identified concerning the uncertainties affecting the r-residuals. Many sr-nuclides are predicted to have a small s-process component only. The r-process contribution to these species, referred to as r-dominant, is clearly quite insensitive to the s-process uncertainties. The situation is just the opposite in the case of s-dominant nuclides.

Some r-process residuals suffer from remarkably large uncertainties, which quite clearly cannot be ignored when discussing the r-process and the virtues of one or another model for this process. This concerns in particular the elements $\mathrm{Rb}, \mathrm{Sr}, \mathrm{Y}, \mathrm{Zr}, \mathrm{Ba}, \mathrm{La}, \mathrm{Ce}$ and $\mathrm{Pb}$. Some of them, and in particular Ba or La, are often used as tracers of the levels of s- or r-processing during the galactic history (see Sect. 9). Lead has also a special status in the studies of the s-process (e.g. Goriely, 2005, for references), as well as of the r-process (see Sect. 9). It could well be of pure s-nature if a strong s-process component can indeed develop in some stars, but a pure r-process origin cannot be excluded. These uncertainties largely blur any picture one might try to draw from observations and from simplistic theoretical considerations.

\section{Isotopic anomalies in the SoS composition}

The bulk SoS composition has been of focal interest since the very beginning of the development of the theory of nucleosynthesis. Further astrophysical interest and excitement have developed with the discovery of the fact that a minute fraction of the SoS material has an isotopic composition deviating from that of the bulk. Such 'isotopic anomalies' are observed in quite a large suite of elements ranging from $\mathrm{C}$ to $\mathrm{Nd}$ (including the rare gases), and are now known to be carried by high-temperature inclusions of primitive meteorites, as well as by various types of meteoritic grains. The inclusions are formed from SoS material out of

\footnotetext{
${ }^{2}$ A MES calculation with the revised solar abundances of Lodders (2010) has not been done, but is expected not to give significantly different results from those reported here
} 
equilibrium with the rest of the solar nebula. The grains are considered to be of circumstellar origin, and to have survived the process of incorporation into the SoS.

Isotopic anomalies contradict the canonical model of an homogeneous and gaseous protosolar nebula, and provide new clues to many astrophysical problems, like the physics and chemistry of interstellar dust grains, the formation and growth of grains in the vicinity of objects with active nucleosynthesis, the circumstances under which stars (and in particular SoS-type structures) can form, as well as the early history of the Sun (in the so-called 'T-Tauri' phase) and of the SoS solid bodies. Last but not least, they raise the question of their nucleosynthesis origin and offer the exciting perspective of complementing the spectroscopic data for chemically peculiar stars in the confrontation between abundance observations and nucleosynthesis models for a very limited number of stellar sources, even possibly a single one. This situation is in marked contrast with the one encountered when trying to understand the bulk SoS composition, which results from the mixture of a large variety of nucleosynthesis events, and consequently requires the modelling of the chemical evolution of the Galaxy.

Among the identified anomalies, several concern the p-, s- and r-nuclides. Those attributed to the p- and r-processes are discussed in some detail by Arnould \& Goriely (2003) and Arnould et al. (2007), and are not reviewed here. As a very brief summary, let us just say that various blends of $\mathrm{p}^{-}, \mathrm{s}-$ and $\mathrm{r}$-nuclides that differ more or less markedly from the bulk SoS mixture depicted in Sect. (2) are identified in a variety of meteorites at various scales, including bulk samples, refractory inclusions or grains viewed from their many highly anomalous isotopic signatures as grains of circumstellar origins. This is generally interpreted in terms of the decoupling between the three mechanisms producing these nuclides, and of the non-uniform mixing of their products. One of the surprises of main relevance in the discussion of the r-process is that those grains that are generally interpreted in terms of supernova (SN) condensates do not carry the unambiguous signature of the r-process that would be expected if indeed SNe are the provileged r-process providers (see Sect. 7).

\section{The r-nuclide content of the Galaxy and its evolution}

In practice, the question of the evolution of the galactic content of the nuclides heavier than iron concerns the s- and r-nuclides only. It is traditionally assumed indeed that the p-nuclides are just as rare in all galactic locations as in the SoS. In such conditions, the p-nuclide abundances outside the SoS are out of reach of spectroscopic studies. On the other hand, the s-process is discussed elsewhere in this volume, so that we focus here only on the evolution of the r-nuclide galactic content.

A substantial observational work has been conducted in recent years on this subject. This effort largely relies on the abundance evolution of $\mathrm{Eu}$, classified as an r-process element on grounds of SoS abundance analyses. The main conclusions derived from this observational work up to 2007 have been discussed in some detail by Arnould et al. (2007). Some new observations have confirmed previous ones, or have brought some interesting new pieces of information which, however, are not considered to bring a real breakthrough in our global understanding of the r-process. Let us just give a very limited flavour of the conclusions that can be drawn from the myriad of available observations:

(i) the Eu data are classically used to support the idea that the r-process has contributed very early to the heavy element content of the Galaxy. However, the observed Eu abundance scatter introduces some confusion when one tries to establish a clear trend of the Eu enrichment with metallicity. It is also difficult to identify the value of $[\mathrm{Fe} / \mathrm{H}]$ at which the signature 
of the s-process becomes identifiable. This conclusion relies in particular on La abundances derived from observation, La being classically considered as an s-element in the SoS (even if a non-negligible r-process contribution cannot be excluded). Recent observations also indicate that star to star variations in the r-process content of metal-poor globular clusters may be a common, although not ubiquitous, phenomenon (Roederer, 2011);

(ii) a most useful information on the relative evolution of the s- and r-process efficiencies in the Galaxy would be provided by the knowledge of the isotopic composition of the neutron-capture elements. Such data are unfortunately very scarce, and concern the Ba isotopic composition in a limited sample of stars. They are still under some debate, but raise the possibility that Ba may be mainly of s-process venue in some metal-poor stars (Gallagher et al. (2010), who refer to this possibility as an 'inconvenient truth');

(iii) much excitement has been raised by the observation that the patterns of abundances of heavy neutron-capture elements between $\mathrm{Ba}$ and $\mathrm{Pb}$ in r-process-rich metal-poor stars are remarkably similar to the SoS one. This claimed 'convergence' or 'universality' has to be taken with some care, however, as it largely relies on the assumption that the decomposition between s- and r-process contributions in metal-poor stars is identical to the SoS one, which has yet to be demonstrated. An interpretation of this universality is proposed in Sect. 6.1;

(iv) no universality appears to hold for $Z \lesssim 58$ and for $Z \gtrsim 76$, where this concerns in particular the Pb-peak elements and the actinides. This situation has far-reaching consequences, particularly regarding the possibility of building galactic chronologies on the actinide content of very metal-poor stars (see Sect. 9);

(v) the different behaviours of the abundance patterns of the elements below and above $\mathrm{Ba}$ have laid the ground for speculations on possible different sites of the r-process. It is discussed by Arnould et al. (2007) that the interest of these speculations is rather limited at the present stage of desperate search for a single suitable site for the r-process (see Sects. 7.2 and 8);

(vi) the knowledge of the r-process content of the galactic cosmic rays (GCRs) is of substantial interest, as they are made of much younger material than the SoS (i.e., about 20 to $30 \mathrm{Myr}$ old). In spite of substantial progress made in the measurement of the GCR composition, much remains to be done. At this time, there is no clear proof that the r-process(es) that has (have) contributed to this recent sample of galactic material is (are) of a similar nature as those responsible for the bulk SoS. The identification of actinides in the GCRs has been made possible recently. An accurate measurement of their abundances would be of great value to discriminate between various GCR sources that have been proposed. As in the stellar case, information on the isotopic composition of the GCRs would be of prime interest in helping to evaluate the fractional contribution of freshly synthesized r-process material to this sample of young galactic material.

\section{Nuclear physics input for the r-process}

It is easy to conceive that the nuclear physics that enters the r-process modelling depends to a more or less large extent on the astrophysics conditions that are suitable for the development of that process. Even if the proper site(s) of the r-process has (have) not been reliably identified yet, it appears reasonable to say from existing studies that a huge body of nuclear data are potentially needed for the purpose of r-process predictions. This includes the static properties (like masses, matter and charge distributions, single-particle spectra, or pairing characteristics) of thousands of nuclides from hydrogen to the superheavy region located 
between the valley of $\beta$-stability and the neutron drip line. Their decay characteristics ( $\alpha$-decay, spontaneous fission, $\beta$-decay, $\beta$-delayed neutron emission or fission), and reactivity (nucleon or $\alpha$-particle captures, photoreactions, or neutrino captures) are needed as well.

A major effort has been devoted in recent years to the measurement of nuclear data of relevance to the r-process. Still, a large body of information remains, and will remain in a foreseeable future, out of reach of experimental capabilities. This is of course the direct consequence of the huge number of nuclear species that may be involved in one r-process or another, along with the fact that nuclei very far from the valley of stability are likely to enter the process. Theory has thus mandatorily to complement the laboratory measurements (see e.g. Dillmann \& Litvinov (2011) and Habs et al. (2011) for a non-exhaustive list of recent laboratory efforts). Predictions based as much as possible on global universal and microscopic models have to be favoured, as discussed in Arnould et al. (2007) where the reader is referred to for many details on the relevant nuclear physics.

\section{The astrophysics of the r-process: parametrized site-free scenarios}

\subsection{Canonical and 'multi-event r-process (MER)' high-temperature models}

Since the early days of the theory of nucleosynthesis, it has been proposed that the r-process results from the availability of neutron concentrations that are so high that neutron captures (especially of the radiative type) are faster than $\beta$-decays, at least for a substantial number of neutron-rich nuclides that are not located too far from the valley of nuclear stability. This is in marked contrast to the situation envisioned for the s-process. Such conditions clearly provide a natural way to transform any pre-existing material into very neutron-rich species, viewed as the progenitors of the r-nuclides. A classical additional hypothesis has been that the otherwise unspecified stellar location is hot enough to allow $(\gamma, \mathrm{n})$ photodisintegrations to counteract to a more or less large extent the action of the inverse radiative neutron captures. Finally, it is supposed that a cooling of the material that allows the 'freezing-out' of the highly temperature-sensitive photodisintegrations occurs concomitantly with a decrease of the neutron density to values that are low enough to freeze the neutron captures. ${ }^{3}$ The hypothesized high neutron fluence and temperature have been the framework adopted by the vast majority of studies of the r-process. In many cases, the consequences of such an assumption have been scrutinized only from a purely nuclear physics point of view, just considering that one astrophysical site or the other, and in particular the inner regions of massive star $\mathrm{SN}$ explosions, could be the required neutron provider.

The simplest and most widely used form of this high-temperature r-process scenario is commonly referred to as the canonical r-process model (see e.g. Arnould et al., 2007, for details). It assumes constant thermodynamic conditions (temperatures, densities, neutron concentrations) during a given irradiation time. Over this period, pre-existing iron-peak material is driven by neutron captures into a location of the neutron-rich region determined by the neutron fluence and by the reverse photodintegrations. When the irradiation stops, all neutron captures and photodisintegrations are abruptly frozen (this implies that the capture of neutrons produced in $\beta$-delayed processes is neglected). Even if this canonical model does not make reference to any specific astrophysics scenario, but builds on nuclear properties only, it has helped greatly paving the way to more sophisticated approaches of the r-process.

\footnotetext{
${ }^{3}$ Let us recall that a transformation is said to be 'frozen' if its typical mean lifetime gets longer than a typical evolutionary timescale of the considered astrophysical site
} 


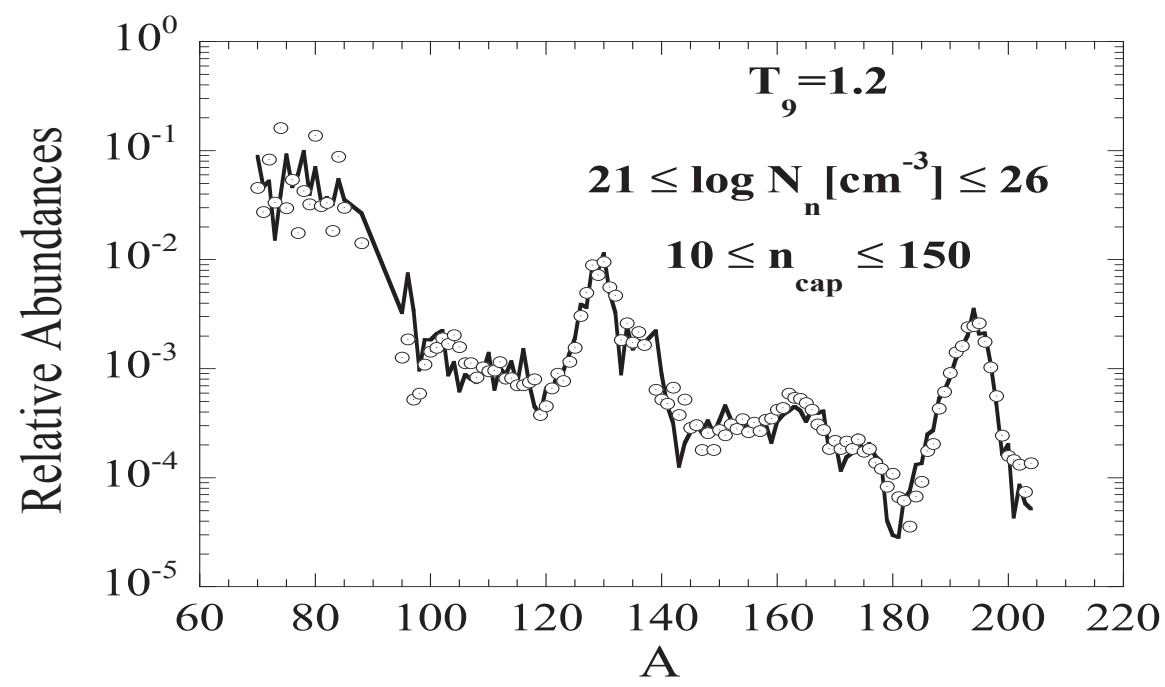

Fig. 5. Comparison between the SoS r-abundances (Fig. 4; the uncertainties are not shown) and a MER fit obtained assuming a constant temperature $T_{9}=1.2$ (in billion $\mathrm{K}$ ) and a superposition of events characterized by free neutron concentrations $N_{\mathrm{n}}$ and numbers of neutrons captured by iron seed $n_{\text {cap }}$ located in the indicated ranges. Within these prescribed limits, the characteristic of the involved events and their relative contributions to the displayed yields are derived from an iterative inversion procedure (see Arnould et al., 2007, for details and references)

A parametric approach of the r-process referred to as the 'multi-event r-process (MER)' has been developed recently (see Arnould et al., 2007, for details). It drops some of the basic assumptions of the canonical model, but keeps the simplification of constant thermodynamic conditions and neutron concentrations during the irradiation time, after which all nuclear reactions are frozen. It is an especially efficient tool to carry out systematic studies of the impact of uncertainties of nuclear physics nature on yield predictions, which, in this approach, necessitate the solution of huge nuclear reaction networks involving thousands of nuclear species, and the availability of an even larger body of input nuclear data (reaction and $\beta$-decay rates, fission probabilities,...). Figure 5 provides an example of fit to the SoS r-abundance distribution obtained with the use of MER.

\subsection{Universality, you have said universality?}

As a special application, MER has been used to interpret the high similarity of the SoS r-abundances between $\mathrm{Ba}$ and Os and those observed in r-process-rich metal-poor stars. This situation is generally interpreted as the signature of a 'universality' of the r-process (see Sect. 4). As reviewed by Arnould et al. (2007), the main conclusions drawn from the MER results are that

(1) the pattern of abundances in the Ba to Os range is mainly governed by nuclear physics properties (and in particular by the fact that even $Z$ elements have more stable isotopes that can be fed by the $r$-process). If this is indeed true, a possible universality in this $Z$ range does not tell 
much about specific astrophysical conditions. It is quite remarkable that this result is largely ignored in the literature on the r-process;

(2) the convergence of abundances in the above mentioned range does not provide any demonstration of any sort of a more global universality involving lighter and heavier elements. With time, these reservations have received mounting support from observation, as noted in Sect. 4.

\subsection{Dynamical high-temperature r-process approaches (DYR)}

In associating the r-process with $\mathrm{SN}$ explosions, several attempts to go beyond the canonical and MER models have been made by taking into account some evolution of the characteristics of the sites of the r-process during its development. These models are coined 'dynamical' (DYR) in the following in order to remind of the time variations of the thermodynamic state of the r-process environment (see Arnould et al., 2007, for references). These models do not rely on any specific explosion scenario. They just assume that a material that is initially hot enough for allowing a nuclear statistical equilibrium (NSE) to be achieved expands and cools in a prescribed way on some selected timescale. This evolution is fully parameterized.

With the requirement of charge and mass conservation, and if the relevant nuclear binding energies are known, the initial NSE composition is determined from the application of the nuclear Saha equation (e.g. Sect. 7-2 of Clayton, 1968, for a general presentation) for an initial temperature and density (or, equivalently, entropy), and electron fraction (net electron number per baryon) $Y_{\mathrm{e}}$. These three quantities are of course free parameters in a site-free r-process approach. The evolution of the abundances during expansion and cooling of the material from the NSE state is derived by solving an appropriate nuclear reaction network. The freeze-out of the charged-particle induced reactions might be followed by an r-process.

With temperature, density and $Y_{\mathrm{e}}$ as free parameters, many choices of initial NSE compositions may clearly be made, involving a dominance of light or heavy nuclides, as illustrated in Fig. 6. However, in view of its relevance to the SN models, an initial NSE at temperatures of the order of $10^{10} \mathrm{~K}$ is generally considered. It favours the recombination of essentially all the available protons into $\alpha$-particles (the region noted NSE $[n, \alpha]$ in Fig. 6). The evolution of this initial composition to the stage of charged-particle induced reaction freeze-out has been analyzed in detail by Meyer et al. (1998)), and we just summarize here some of its most important features that are of relevance to a possible subsequent r-process:

(1) at some point in the course of the expansion and cooling of the initially $\alpha$-rich material, full NSE breaks down as the result of the slowness of a fraction of the charged-particle reactions relative to the expansion timescale. The formation of quasi-equilibrium (QSE) clusters results. In this state, the intra-QSE composition still follows the NSE Saha equation, but the relative inter-cluster abundances do not, and depend on the kinetics of the nuclear flows into and out of the QSE clusters. To be more specific, the QSE phase is dominated in its early stages by a light cluster made of neutrons, $\alpha$-particles and traces of protons, and by a cluster made of ${ }^{12} \mathrm{C}$ and heavier species. The population of the latter is determined mainly by the $\alpha+\alpha+n$ reaction, followed by ${ }^{9} \mathrm{Be}(\alpha, n){ }^{12} \mathrm{C}(n, \gamma){ }^{13} \mathrm{C}(\alpha, n){ }^{16} \mathrm{O}$, as first noticed by Delano \& Cameron (1971);

(2) as the temperature decreases further, the QSE clusters fragment more and more into smaller clusters until total breakdown of the QSE approximation, at which point the abundances of all nuclides have to be calculated from a full nuclear reaction network. In the 


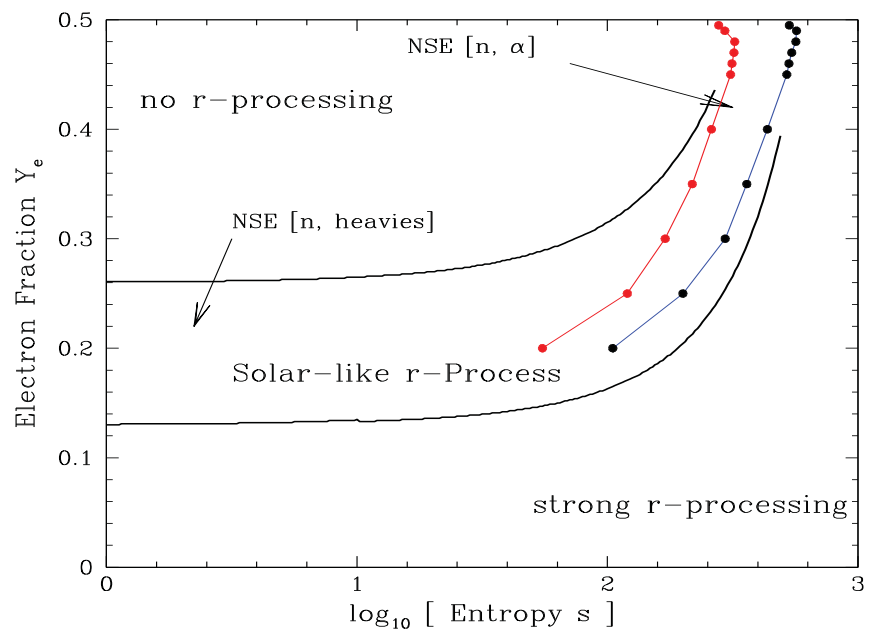

Fig. 6. The likelihood of a DYR r-process for given combinations of the electron fraction $Y_{\mathrm{e}}$ and the entropy per baryon $s$. A SoS-like r-process is expected for a suitable superposition of conditions between the black lines. The results inferred from an initial NSE phase at low $s$ are smoothly connected to those of various nuclear network calculations for high $s$ values. In the latter cases, the assumed expansion timescales imply that the charged-particle induced reaction freeze-out is reached after dynamical timescales $\tau_{\mathrm{d} y n}$ in excess of about $50-100 \mathrm{~ms}$. The two dotted lines represent the contours of successful r-processing for $\tau_{\mathrm{d} y n}=50 \mathrm{~ms}$ (left line) and $100 \mathrm{~ms}$ (right line) (see Hoffman et al., 1997, for details)

relevant $\alpha$-particle-rich environment, the reaction flows are dominated by $(\alpha, \gamma)$ and $(\alpha, n)$ reactions with the addition of radiative neutron captures. Nuclei as heavy as Fe or even beyond may result. For a low enough temperature, all charged-particle-induced reactions freeze-out, only neutron captures being still possible. This freeze-out is made even more efficient if the temperature decrease is accompanied with a drop of the density $\rho$, which is especially efficient in bringing the operation of the $\rho^{2}$-dependent $\alpha+\alpha+n$ reaction to an end. In the following, the neutron-rich $\alpha$-rich process summarized above will be referred to as the $\alpha$-process for simplicity, and for keeping the terminology introduced by Woosley \& Hoffman (1992).

The composition of the material at the time of freeze-out depends on the initial $Y_{\mathrm{e}}$, on the entropy s (see Meyer et al., 1998, for a detailed discussion), as well as on the dynamical timescale $\tau_{\mathrm{d} y n}$. The heavy nuclei synthesized at that moment may have on average neutron numbers close to the $N=50$ closed shell, and an average mass number around $A=100$. These nuclei can be envisioned to be the seeds for a subsequent r-process, in replacement of the iron peak assumed in the canonical and MER models. For a robust r-process to develop, favourable conditions have to be fulfilled at the time of the $\alpha$-process freeze-out. In particular, the ratio at that time of the neutron concentration to the abundance of heavy neutron-rich seeds has to be high enough for allowing even the heaviest r-nuclides to be produced. As an example, $A=200$ nuclei can be produced if an average of 100 neutrons are available per $A=100$ nuclei that could emerge from the $\alpha$-process. The availability of a large enough number of neutrons per seed can be obtained under different circumstances: (i) at high enough 
entropies (high enough temperatures and low enough densities), even in absence of a large neutron excess, as it is the case if $Y_{\mathrm{e}}$ is close to 0.5 (Woosley \& Hoffman, 1992), (ii) at lower entropies if $Y_{\mathrm{e}}$ is low enough, and/or (iii) if the temperature decrease is fast enough for avoiding a too prolific production of heavy seeds. Figure 6 sketches in a semi-quantitative way the conclusions of the discussion above concerning the likelihood of development of a successful r-process in terms of entropy and $Y_{\mathrm{e}}$.

\subsection{A high-density r-process scenario (HIDER)}

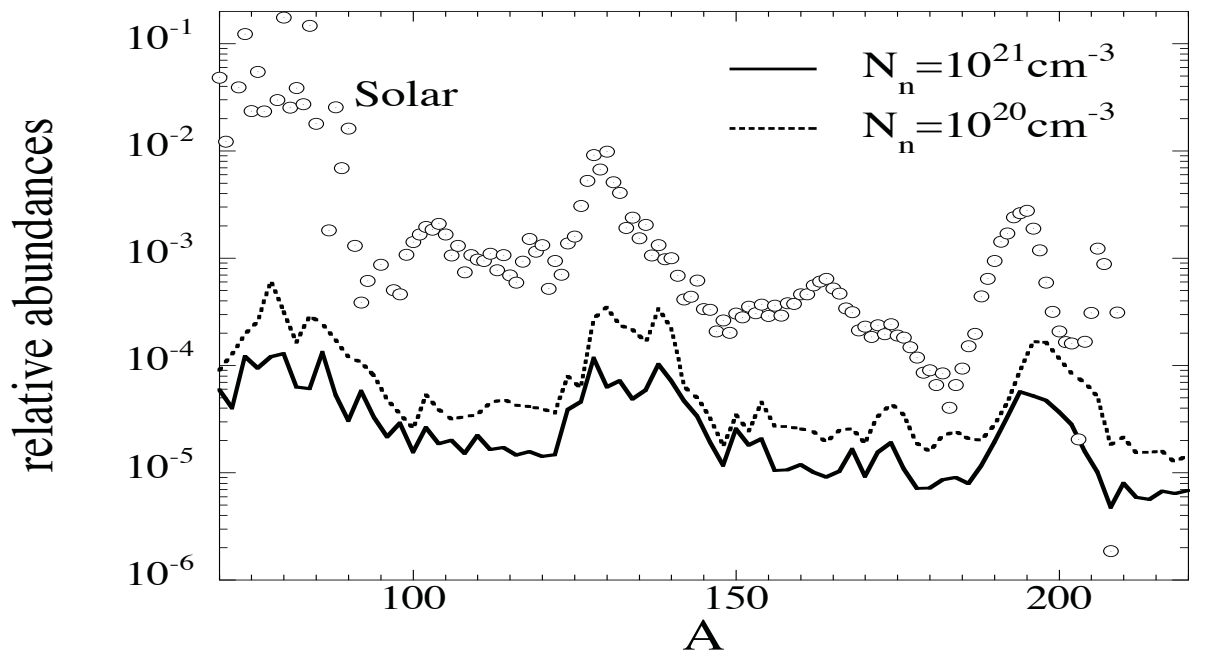

Fig. 7. Abundance distributions predicted by the steady flow HIDER for $N_{n}=10^{20}$ and $10^{21} \mathrm{~cm}^{-3}$. The details of the adopted nuclear physics can be found in Arnould et al. (2007). The SoS abundances are shown for illustrative purpose

Early in the development of the theory of nucleosynthesis, an alternative to the high- $T$ r-process canonical model has been proposed (Tsuruta \& Cameron, 1965). It relies on the fact that very high densities (say $\rho>10^{10} \mathrm{gcm}^{-3}$ ) can lead material deep into the neutron-rich side of the valley of nuclear stability as a result of the operation of endothermic free electron captures. This so-called 'neutronization' of the material is possible even at the $T=0$ limit. The astrophysical plausibility of this scenario in accounting for the production of the r-nuclides has long been questioned, and has remained largely unexplored until the study of the composition of the outer and inner crusts of neutron stars (Baym et al., 1971) and of the decompression of cold neutronized matter resulting from tidal effects of a black hole on a neutron star companion (Lattimer et al., 1977). The decompression of cold neutron star matter has been studied further recently (Sect. 8).

In view of the renewed interest for a high-density r-process, a simple steady flow model, referred to in the following as HIDER, may be developed. Irrespective of the specific details of a given astrophysical scenario, it allows to follow in a very simple and approximate way the evolution of the composition of an initial cold (say $T=0$ ) highly neutronized matter under the combined effect of $\beta$-decays and of the captures of free neutrons that are an important initial component of the considered material. These are the only two types of transformations that 
have to be considered if fissions are disregarded, and if any heating of the material resulting from the $\beta$-decay energy deposition is neglected, so that photodisintegrations can be ignored.

The predictions of HIDER under the additional assumption of a steady flow $(\mathrm{d} N(A) / \mathrm{d} t=0$, $N(A)$ being the total abundance of all the isobars with mass number $A$; see Arnould et al. (2007) for details) are illustrated in Fig. 7. This model does roughly as well in reproducing the three SoS abundance peaks as a steady state high- $T$ canonical model for comparable neutron densities. In other words, a high- $T$ environment is not a necessary condition to account either for the location, or for the width of the observed SoS r-abundance peaks.

\section{Supernovae: a high-temperature site for the r-process, really?}

\subsection{The current status of supernova simulations: a brief review}

As recalled above, the r-process has for long been associated with $\mathrm{SN}$ explosions. Over the years, many attempts have been conducted to better characterize the SN site where an r-process can develop. One has to acknowledge that these efforts have not been really successful up to now. Even simulating the explosion itself represents a real nightmare, as we briefly review below (see Arnould et al., 2007, for additional details and references).

At the end of their nuclear evolution, stars between about 10 and $100 M_{\odot}$ develop a core made of nuclides of the iron group ('iron core') at temperatures in excess of about $4 \times 10^{9} \mathrm{~K}$. As these nuclides have the highest binding energy per nucleon, further nuclear energy cannot be released at this stage, so that the iron core contracts and heats up. This triggers endothermic photodisintegrations of the iron-group nuclides down to $\alpha$-particles, and even nucleons. The corresponding energy deficit is accompanied with a pressure decrease which can be responsible of the acceleration of the contraction into a collapse of the core. Endothermic electron captures can make things even worse. To a first approximation, this gravitational instability sets in near the classical Chandrasekhar mass limit for cold white dwarfs, $M_{\mathrm{Ch}}=$ $5.83 Y_{\mathrm{e}}^{2}$ (as above, $Y_{\mathrm{e}}$ is the electron mole fraction).

The gravitational collapse of the iron core does not stop before the central densities exceed the nuclear matter density $\rho_{0} \approx 2.5 \times 10^{14} \mathrm{~g} \mathrm{~cm}^{-3}$ by about a factor of two. At this point, the innermost $\left(M \lesssim 0.5 M_{\odot}\right)$ material forms an incompressible, hot and still lepton-rich 'proto-neutron' star (PNS) whose collapse is stopped abruptly. A shock wave powered by the gravitational binding energy released in the collapse propagates supersonically into the infalling outer layers. For many years there has been hope that this shock could be sufficiently strong for ejecting explosively most of the material outside the core, producing a so-called 'prompt core collapse supernova' (PCCSN) with a typical kinetic energy of $1-2 \times 10^{51}$ ergs, as observed. The problem is that the shock is formed roughly half-way inside the iron core, and looses a substantial fraction of its energy in the endothermic photodisintegrations of the iron-group nuclei located in the outermost portion of the core. The shock energy loss is aggravated further by the escape of the neutrinos produced by electron captures on the abundant free protons in the shock-heated material. Detailed one-dimensional hydrodynamic simulations conclude that the initially outgoing shock wave transforms within a few milliseconds after bounce into an accretion shock. The matter behind the shock continues to accrete on the PNS. The bottom line is that no recent simulation is able to predict a successful PCCSN for a Fe-core progenitor star $\left(M \gtrsim 10 M_{\odot}\right)$.

Even so, some hope to get a CCSN of a non-prompt type has been expressed if there is a way to 'rejuvenate' the shock efficiently enough to obtain an explosive ejection of the material outside 
the PNS. This rejuvenation remains a matter of intensive research. Neutrinos might well play a pivotal role in this matter. They are produced in profusion from the internal energy reservoir of the PNS that cools and deleptonizes hundreds of milliseconds after bounce, and their total energy might amount to several $10^{53}$ ergs, that is about 100 times the typical explosion energy of a Type II supernova (SNII). The deposition of a few percent of this energy would thus be sufficient to unbind the stellar mantle and envelope, and provoke a 'delayed' CCSN (DCCSN) (these qualitative statements assume that a black hole is not formed instead of a PNS; see below). Very many attempts to evaluate the precise level of neutrino energy deposition have been conducted over the last decades, based on more or less controversial simplifications of the treatment of the neutrino transport (e.g. Liebendorfer et al., 2005, for a recent re-analysis of the problem, which is made even more complex by the due consideration of neutrino flavour mixing). In fact, theoretical investigations and numerical simulations performed with increasing sophistication over the past 20 years have not been able to come up with a clearly successful DCCSN for a Fe-core progenitor. This conclusion is apparently robust to changes in the highly complex physical ingredients (like the neutrino interactions, or the equation of state), and in the numerical techniques (e.g. Liebendorfer et al., 2005). In fact, the neutrino energy deposition should have to be significantly enhanced over the calculated one in order to trigger an explosion.

This adverse situation may not mark the end of any hope to get a DCCSN, however. In the case of single stars considered here, one might just have to limit the considerations to stars in the approximate 9 to $10 M_{\odot}$ range. These stars possibly develop O-Ne cores instead of iron cores at the termination of their hydrostatic evolution. Efficient endothermic electron captures could trigger the collapse of that core, which could eventually transform into a so-called electron-capture SN that may be of the SNII or Type Ia (SNIa) type, depending upon the extent of the pre-explosion wind mass losses (binary systems might offer additional opportunities of obtaining electron-capture $\mathrm{SNe}$, as mentioned below). Just as in the case of more massive stars, a PCCSN is not obtained. However, a successful DCCSN is predicted (Kitaura et al., 2006). The neutrino heating is efficient enough for rejuvenating the shock wave about $150 \mathrm{~ms}$ after bounce, and mass shells start being ablated from the PNS surface about $50 \mathrm{~ms}$ later, leading to a so-called 'neutrino-driven wind' ${ }^{4}$ No information is provided by the current simulations on the conditions at times much later than a second after bounce. Note that the predicted successful delayed electron capture SN is characterized by a low final explosion energy (of the order of $10^{50}$ ergs, which is roughly ten times lower than typical SN values), and by just a small amount of ejected material (only about $0.015 M_{\odot}$ ). These features might suggest a possible connection with some subluminous SNII events and with the Crab nebula.

Note that the outcome of a failed CCSN is the transformation of the PNS into a black hole through the fallback onto the neutron star of the material that cannot be shock ejected. A black hole is even expected to form 'directly' instead by fallback in $M \gtrsim 40 M_{\odot}$ non-rotating stars, at least under the assumption of no strong mass losses. In fact, this assumption is likely to be invalid for a large fraction at least of the not too low metallicity $M \gtrsim 40 M_{\odot}$ stars which transform through strong steady mass losses into Wolf-Rayet stars that might eventually experience a DCCSN of the SNIb/c type.

A major effort has been put recently in the development of simulations of explosions that go beyond the one-dimensional approximation. This is motivated not only by the difficulty of obtaining successful CCSNe in one-dimensional simulations, as briefly reviewed above,

\footnotetext{
${ }^{4}$ Unless otherwise stated, neutrino-driven winds refer to transonic as well as subsonic winds. In case one has to be more specific, a subsonic wind is referred to as a breeze
} 
but also by the mounting observational evidence that SN explosions deviate from spherical symmetry, not talking about the possible connection between the so-called soft long-duration gamma-ray bursts and grossly asymmetric explosions accompanied with narrow jets of relativistic particles, referred to as JetSNe. The multi-dimensional extension of the simulations opens the potentiality to treat in a proper way different effects that may turn out to be essential in the CCSN or JetSNe process. They include fluid instabilities, rotation and magnetic fields, or, possibly, acoustic power triggering (see Arnould et al., 2007, for more details and references). These effects come of course on top of the neutrino transport already built into the one-dimensional models. The exact properties of this transport remain a subject of active research. In particular, the role of collective neutrino oscillations on the SN explosion has been studied recently by Duan \& Friedland (2011). They consider that this phenomenon still needs a more complete physical understanding, and still has a rich potential of surprises.

\subsection{Neutrino-driven winds and the r-process}

The neutrino-driven winds that may accompany successful DCCSNe are certainly interesting from a purely hydrodynamical point of view, even if they may turn out not to be the key triggering agents of DCCSNe. In addition, their nucleosynthesis has been scrutinized in detail, especially following the excitement raised by the hope that they could provide a natural site for an $\alpha$-process and for a subsequent dynamical r-process (Sect. 6.3, and e.g. Takahashi et al. (1994) for early calculations). This hope has gained support from a one-dimensional DCCSN simulation of an iron-core progenitor predicting that entropies as high as about 400 could be attained in the wind more than 10 seconds after bounce (Woosley et al., 1994). Such a high entropy allows the development of a robust r-process for a large variety of values of the neutron excess or $Y_{\mathrm{e}}$ and dynamical timescale $\tau_{\mathrm{dyn}}$ (see Sect. 6.3 and Fig. 6). However, another one-dimensional iron-core DCCSN model has predicted about five times lower entropies, so that the development of an extended r-process is severely endangered (Takahashi et al., 1994). The subsequent studies have confirmed that this r-process scenario could only be recovered at the expense of some twists that are difficult to justify in general (e.g. Qian \& Woosley, 1996) and that large late time entropies (Woosley et al., 1994) were unrealistic due to some problems with the equation of state (Roberts et al., 2010). As a consequence, recent studies conclude that the simplest model of the neutrino-driven wind can most likely not produce the r-process (Roberts et al., 2010).

In such an unsatisfactory state of affairs, the best one can do is to try to understand better the physics of neutrino-driven winds through the development of (semi-)analytical models some aspects of which may be inspired by (failed) explosion simulations, and to try to delineate on such grounds favourable conditions for the development of the r-process. These analytical models confirm that the wind nucleosynthesis depends on $Y_{\mathrm{e}}$, entropy $s$, and $\tau_{\text {dyn }}$ as in the $\alpha$-process discussed in Sect. 6.3. The wind mass-loss rate $\dot{M}$ is influential as well. Ultimately, the quantities acting upon the synthesis in the neutrino-driven DCCSN model depend crucially on the details of the interaction of neutrinos with the innermost SN layers, as well as on the mechanisms that might aid to get a successful DCCSN, and whose relative importance remains to be quantified in detail.

Several wind models of analytical nature exist. They differ in their level of physical sophistication and in their way to parametrize the wind characteristics. In all cases, the wind is assumed to be spherically symmetric, which appears to be a reasonable first approximation even in two-dimensional simulations, at least late enough after core bounce. In addition, the wind is generally treated as a stationary flow, meaning no explicit time dependence of any 
physical quantity at a given radial position. Newtonian and post-Newtonian descriptions of a spherically symmetric stationary neutrino-driven (supersonic) wind or (subsonic) breeze emerging from the surface of a PNS have been developed. The reader is referred to Arnould et al. (2007) for the presentation of a Newtonian, adiabatic and steady-state model for the wind and breeze regimes, and for a general-relativistic steady-state wind solution.

A comment is in order here concerning the relevance of the wind or breeze regimes. Is one of the two favoured by the DCCSN physics? This question is far from being just academic, as it is likely that its answer may have some impact on the predicted development of the r-process. It is quite intricate as well. One difficulty arises as the neutrino-driven material is likely not to flow unperturbed to infinity in a variety of DCCSN situations. The wind may in particular interact with matter and radiation in that portion of the star through which the SN shock has already passed. This interaction is likely to depend, among other things, on the pre-SN structure. As an example, it is clearly more limited as the mass of the outer layers decreases when going from massive SNII progenitors to SNIb/c events whose progenitors (Wolf-Rayet stars) have lost their extended $\mathrm{H}$-rich envelope prior to the explosion. The interaction of the material ablated from the PNS and the outer SN layers has several important consequences. It may give rise to a reverse shock responsible for the fallback of a more or less large amount of material onto the PNS, and whose properties (location and strength) alter more or less deeply the characteristics of the neutrino-ejected material. For large enough energies of the reverse shock, the wind may indeed transform into a breeze.

Figure 8 displays the evolution of temperature and density for a particular breeze solution discussed in Arnould et al. (2007). Some snapshots of the corresponding progressive build-up of heavy nuclei by the $\alpha$-process, followed by the production of r-nuclides are shown in Fig. 9 . The r-nuclide abundance distributions calculated for a breeze solution that is slightly different from the one displayed in Fig. 8 (even if the PNS mass and entropy are the same; see Arnould et al., 2007, for details) and three different mass loss rates (leading to three different cooling timescales) are shown in Fig. 10. The influence on the $r$-abundances of different values of other breeze parameters is discussed by Arnould et al. (2007).

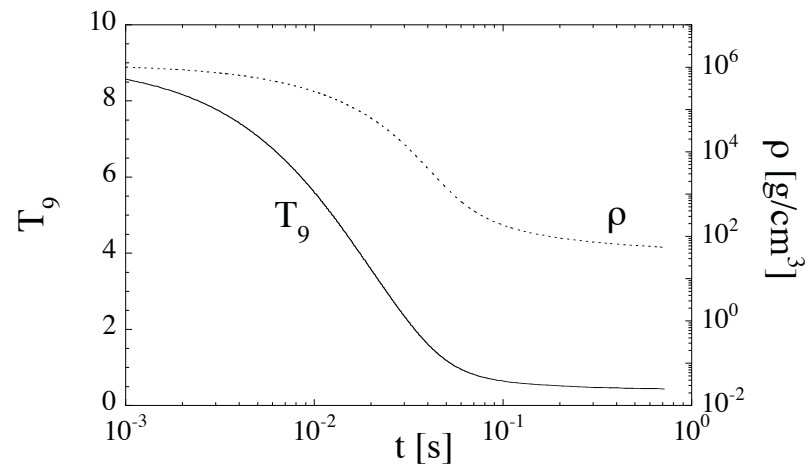

Fig. 8. Evolution of density $\rho$ and of temperature $T_{9}$ (in $10^{9} \mathrm{~K}$ ) calculated in the Newtonian approximation for a breeze starting about $14 \mathrm{~km}$ away from a $1.5 \mathrm{M}_{\odot}$ PNS with a temperature $T_{9}=9$. The displayed solution depends on other parameters (including entropy and mass loss rate) that are discussed in Arnould et al. (2007) 


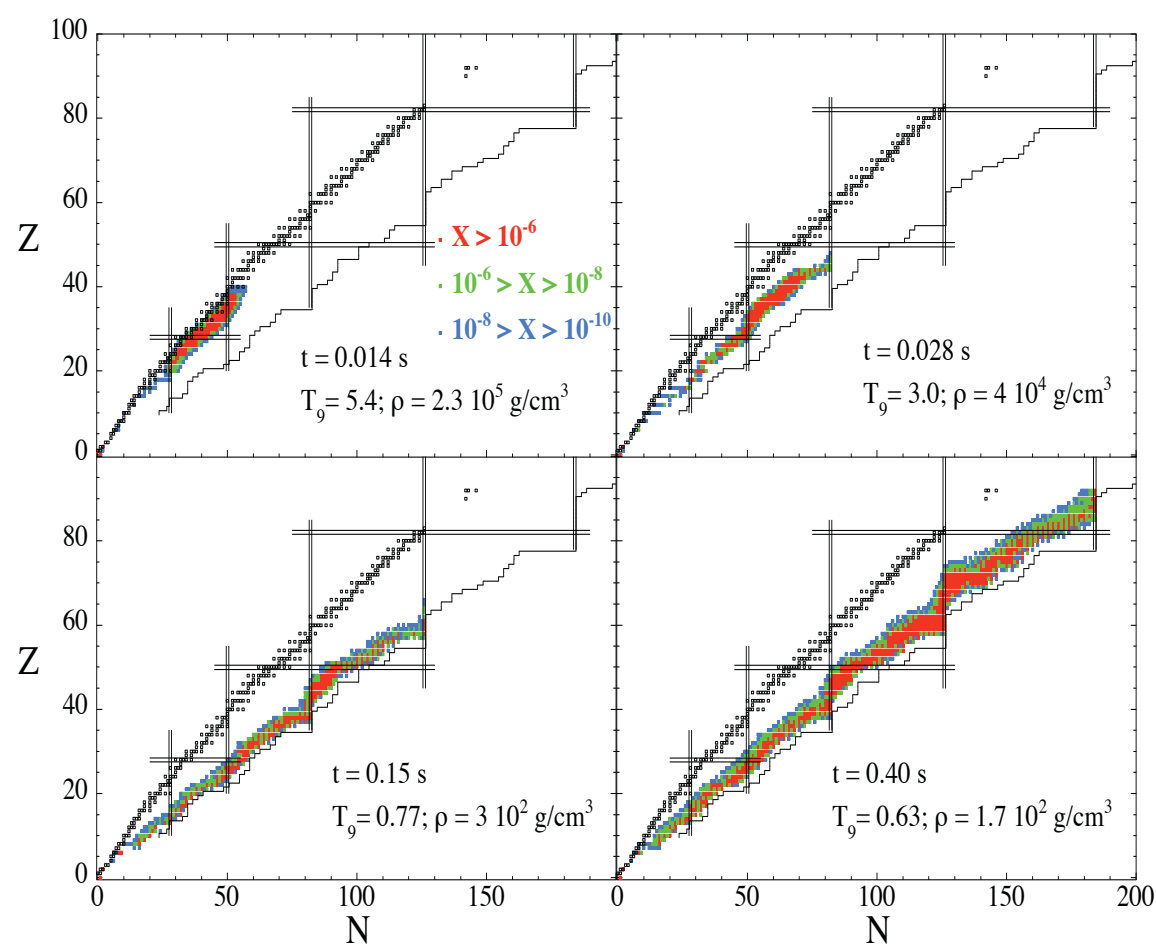

Fig. 9. Snapshots of the nuclear flows calculated for the breeze solution of Fig. 8. The first three panels $(t \leq 0.15 \mathrm{~s}$ ) describe the progressive build-up of heavy nuclei by the $\alpha$-process. These act as the seeds for the r-process that develops after the $\alpha$-process freeze-out, as shown in the last panel

The influence of the neutrino luminosity on the r-process is also reviewed by Arnould et al. (2007). The neutrino interaction is detrimental to the r-process as a result of the reduction of the number of neutrons available per seed nucleus. In addition, the abundance distribution is reshaped by the neutrino interactions. Such effects strongly depend on the adopted neutrino luminosities and temperatures, which remain rather uncertain. The impact of neutrinos on the r-process has been further explored recently (Duan \& Friedland, 2011; Duan et al., 2011). They demonstrate that neutrino flavour transformations, and more specifically collective oscillations, might play a role in the $\mathrm{SN}$ explosion and might decrease further the r-process yields.

Let us finally report on some recent work concerning

(1) the possible development of an r-process in electron-capture SNe studied in 1D (Wanajo et al., 2009) and 2D (Wanajo et al., 2011) simulations. These models show that some neutron-rich material can be expelled. In particular, the $2 \mathrm{D}$ calculations predict the ejection of lumps of material enriched with some r-nuclides up to about $\mathrm{Zr}$ that are reminiscent of the abundance patterns in some r-nuclide deficient halo stars. However, the possibility of development of 


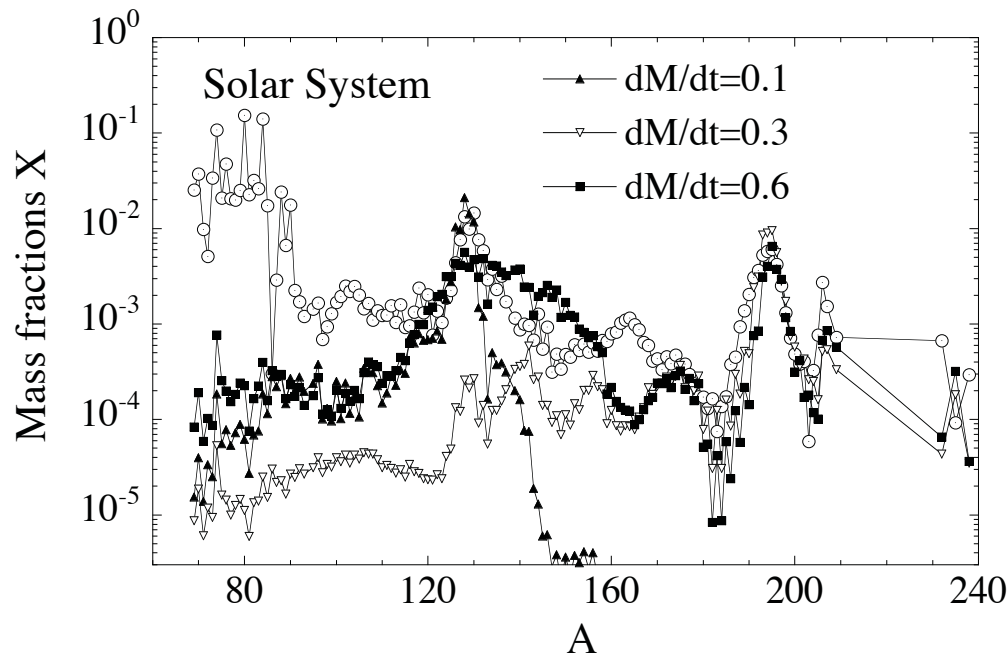

Fig. 10. Distribution of the r-nuclide abundances derived for a breeze solution obtained with a PNS mass of $1.5 M_{\odot}$, an initial electron fraction $Y_{\mathrm{e}}=0.48$, and different values of the mass loss rate $d M / d t$ (in units of $10^{-5} M_{\odot} / \mathrm{s}$ ). The open dots give the SoS r-nuclide abundances normalized to $\sum_{i} X_{i}=1$. See Arnould et al. (2007) for details, particularly on the selected breeze solution

a limited r-process in electron-capture SNe remains to be confirmed by more reliable pre-SN models and by 3D simulations with high enough resolution;

(2) a further examination of the possibility of r-nuclide production in the He-shell of massive stars. As reviewed by Arnould et al. (2007), a neutron-capture episode could be encountered in explosive He-burning as a result of the neutrons produced by $(\alpha, n)$ reactions on pre-existing ${ }^{22} \mathrm{Ne}$ or $\mathrm{Mg}$ isotopes. This neutron supply is, however, found to be so weak that it could only lead to a limited redistribution of pre-explosion heavy nuclide abundances. It has been speculated that some meteoritic r-nuclide anomalies could be generated this way. The $(\alpha, n)$ neutron production could be augmented by neutral (Epstein et al., 1988) or charged-current (Banerjee et al., 2011) neutrino reactions on ${ }^{4} \mathrm{He}$, the necessary neutrinos streaming out of the PNS at the centre of the exploding star. It is concluded by Banerjee et al. (2011) that r-nuclide abundance peaks at $A=130$ and 195 could be expected in very metal-poor $\left(Z \lesssim 10^{-3} Z_{\odot}\right)$ stars.

\section{Compact objects: a site for the high-density r-process scenario?}

As reminded in Sect. 6.4, the decompression of the crust of cold neutron stars (NSs) has long been envisioned as a possible site for the development of a high-density r-process (HIDER). Recently, special attention has been paid to the coalescence of two NSs or of a NS and a BH. This interest follows the confirmation by hydrodynamic simulations that a non-negligible amount of matter can be ejected in such events (Janka et al., 1999; Oechslin et al., 2007; Rosswog et al., 2004), and that this material should be enriched in r-nuclides (Freiburghaus et al., 1999; Goriely, 2005). The ejection of initially cold, decompressed NS matter might also occur in other astrophysical scenarios like giant flares in soft-gamma repeaters, the explosion 
of a NS eroded below its minimum mass, or the equatorial shedding of material from very rapidly rotating supramassive or ultramassive NSs (see Arnould et al., 2007, for more details).

Little effort has so far been devoted to determining the composition of the matter that undergoes a decompression from initially cold NS crust conditions. The first detailed calculation was performed by Meyer (1989) in a systematic parametric study, but only the decompression down to densities around the neutron drip density $\rho_{\text {drip }} \simeq 4.2 \times 10^{11} \mathrm{~g} / \mathrm{cm}^{3}$ was followed. Most of the subsequent investigations of the ejecta from coalescing NSs (Arnould et al., 2007; Freiburghaus et al., 1999; Goriely, 2005; Metzger et al., 2010; Roberts et al., 2011) were parametrized in one way or another, which makes their results and conclusions open to questions. In Goriely (2005) and Arnould et al. (2007) the thermodynamic profiles were constructed from a simple decompression model (see Goriely et al., 2011), but the neutron enrichment (or equivalently the electron fraction $Y_{e}$ ) was consistently taken from $\beta$-equilibrium assumed to have been achieved at the initial density prior to the decompression. It was found, as shown in Fig. 11, that the final composition of the material ejected from the inner crust depends on the initial density, at least for the outer parts of the inner crust at $\rho_{\text {drip }} \leq \rho \leq 10^{12} \mathrm{~g} / \mathrm{cm}^{3}$. For the deeper inner crust layers $\left(\rho>10^{12} \mathrm{~g} / \mathrm{cm}^{3}\right)$, large neutron-to-seed ratios drive the nuclear flow into the very heavy-mass region, leading to multiple fission recycling. As a consequence, the resulting abundance distribution becomes independent of the initial conditions, especially of the initial density. It was found to be in close agreement with the solar distribution for $A>140$ nuclei, as shown in Fig. 12 (Arnould et al., 2007; Goriely, 2005).

Different approaches were taken by Freiburghaus et al. (1999); Metzger et al. (2010) and Roberts et al. (2011). In their calculations, while the density evolution of the mass elements was adopted from hydrodynamical simulations, both the initial neutron enrichment and the temperature history were taken as free parameters. In particular, $Y_{e}$ was chosen in order to obtain, after decompression, an r-abundance distribution as close as possible to the solar distribution. This led to values of $Y_{e}=0.1$ (Freiburghaus et al., 1999) or 0.2 (Roberts et al., 2011), corresponding to relatively near-surface layers of the inner crust and to nuclear flows that are not subject to multiple fission cycles.

Recent nucleosynthesis calculations (Goriely et al., 2011) have performed in the framework of the hydrodynamic simulation of a double $1.35 M_{\odot}$ and of a 1.20-1.50 $M_{\odot}$ NS binary, where about $3 \times 10^{-3}$ and $6 \times 10^{-3} M_{\odot}$, respectively, of the system is found to become gravitationally unbound. In contrast to previous studies, detailed information about the density, $Y_{e}$, and entropy evolution of the ejecta is extracted from the hydrodynamical simulations and included in the network calculations. The ejected mass elements (referred to as 'particles') originate essentially from the inner crust, and more precisely from two different layers at densities below and above roughly $0.3 \times \rho_{S}$ (where $\rho_{S}$ is the nuclear saturation density), as shown in the density histogram of Fig. 13. The low-density set of surface particles is ejected through tidal forces without being much affected by the coalescence. When the NSs come in contact for the first time, some particles deeper in the inner crust are shocked and ejected perpendicularly to the orbital plane. These particles have a higher initial density $\left(\rho \gtrsim 0.4 \rho_{S}\right)$ than the crust particles ejected by tidal forces. Both types of expelled particles have a low initial electron fraction ranging between 0.015 and 0.050 (see Fig. 13, where $Y_{e}=0.015$ in about $45 \%$ of the mass).

As far as the temperature history is concerned, most of the particles are heated during the ejection process to temperatures above $1 \mathrm{MeV}$. At these temperatures, a particle has a NSE composition at its own density and $Y_{e}$. At the time the drip density is reached, 


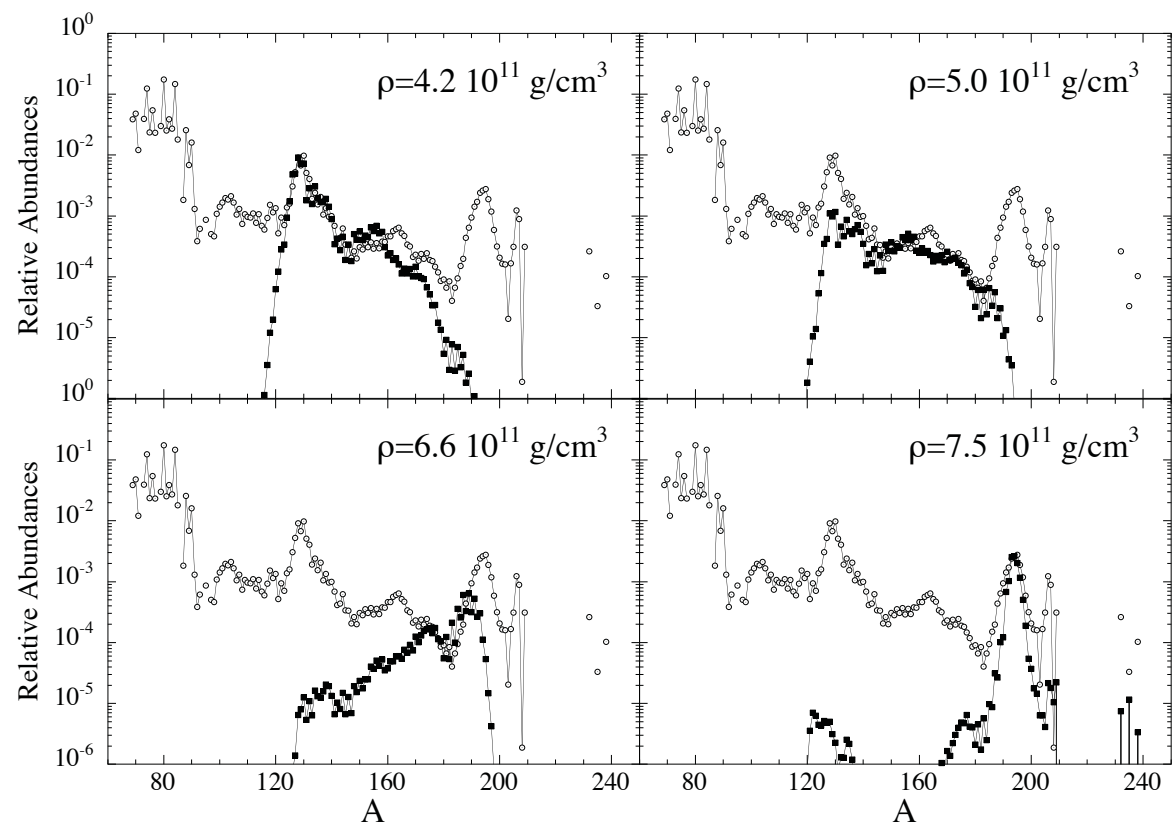

Fig. 11. Composition of the ejected NS inner crust material with four different initial densities lower than $10^{12} \mathrm{~g} / \mathrm{cm}^{3}$ (black squares). The abundances are normalized to the SoS r-abundance distribution shown by open dots (from Arnould et al., 2007)

most of the ejected matter has cooled below $1 \mathrm{MeV}$ (see Fig. 13, right panel) and the NSE has frozen out. As soon as the particle temperature has dropped below $10^{10} \mathrm{~K}$, the particle composition, initially at NSE, must be followed by a full network calculation at the temperatures determined on the basis of the laws of thermodynamics allowing for possible nuclear heating through $\beta$-decays, fission and $\alpha$-decay processes, as described in Meyer (1989).

In this specific r-process scenario, the neutron density is initially so high $\left(N_{n} \simeq 10^{33-35} \mathrm{~cm}^{-3}\right)$ that the nuclear flow follows for the first hundreds of $\mathrm{ms}$ a path licking the neutron drip line. For $Z \geq 103$, fission becomes efficient and recycling takes place two to three times before the neutrons are totally exhausted, as shown in Fig. 14 by the oscillating behaviour of the time evolution of the charge $\langle Z\rangle$ and mass number $\langle A\rangle$ mass-averaged over all the ejected particles. After several hundreds of ms, the density has dropped by a few orders of magnitude and the neutron density experiences a dramatic fall-off as a result of the neutron exhaution by captures. During this period of time, the nuclear flow around the $N=126$ region follows an isotonic chain. When the neutron density reaches some $N_{n}=10^{20} \mathrm{~cm}^{-3}$, the timescale of neutron captures by the most abundant $N=126$ nuclei becomes longer than a few seconds, and the nuclear flow is dominated by $\beta$-decays back to the stability line (as well as by fissions and $\alpha$-decays for the heaviest species). During the decompression, the average temperature remains rather low (around $T_{9}=0.3-0.5 ;$ Fig. 14), so that photoreactions do not play a major role.

The final mass-averaged composition of the ejected particles is shown in Fig. 15. The $A=195$ abundance peak related to the $N=126$ shell closure is produced in SoS distribution, and 


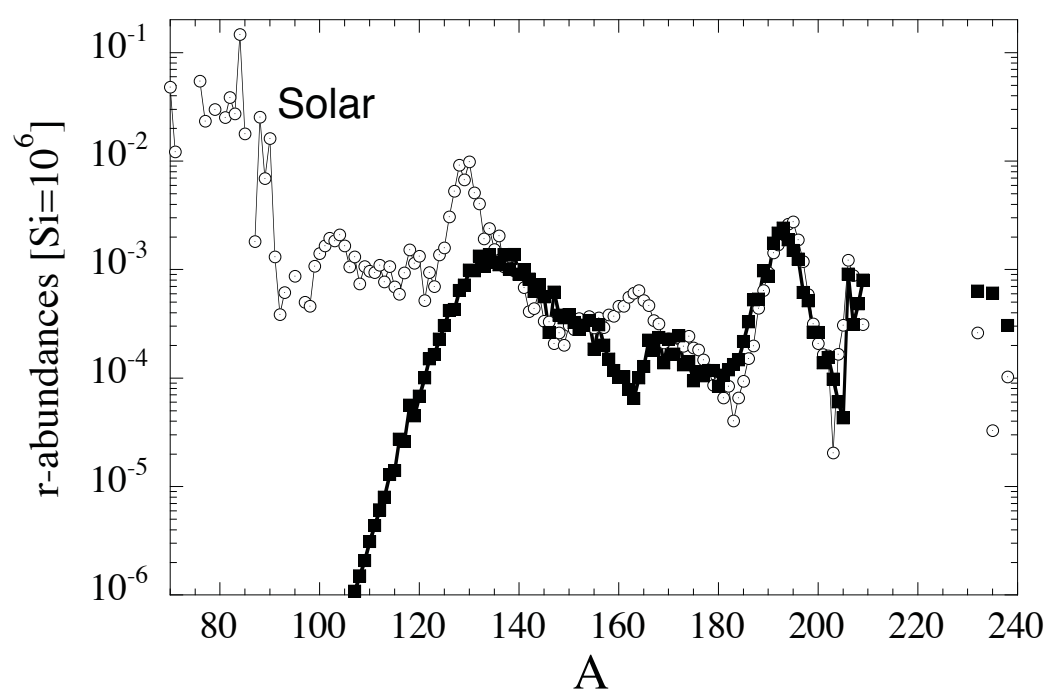

Fig. 12. Final r-abundance distribution (black squares) for a clump of material with initial density $\rho=10^{14} \mathrm{~g} / \mathrm{cm}^{3}$ expanding on a timescale $\tau_{\text {exp }}=6.5 \mathrm{~ms}$. The SoS r-abundance distribution is also shown (from Goriely, 2005)
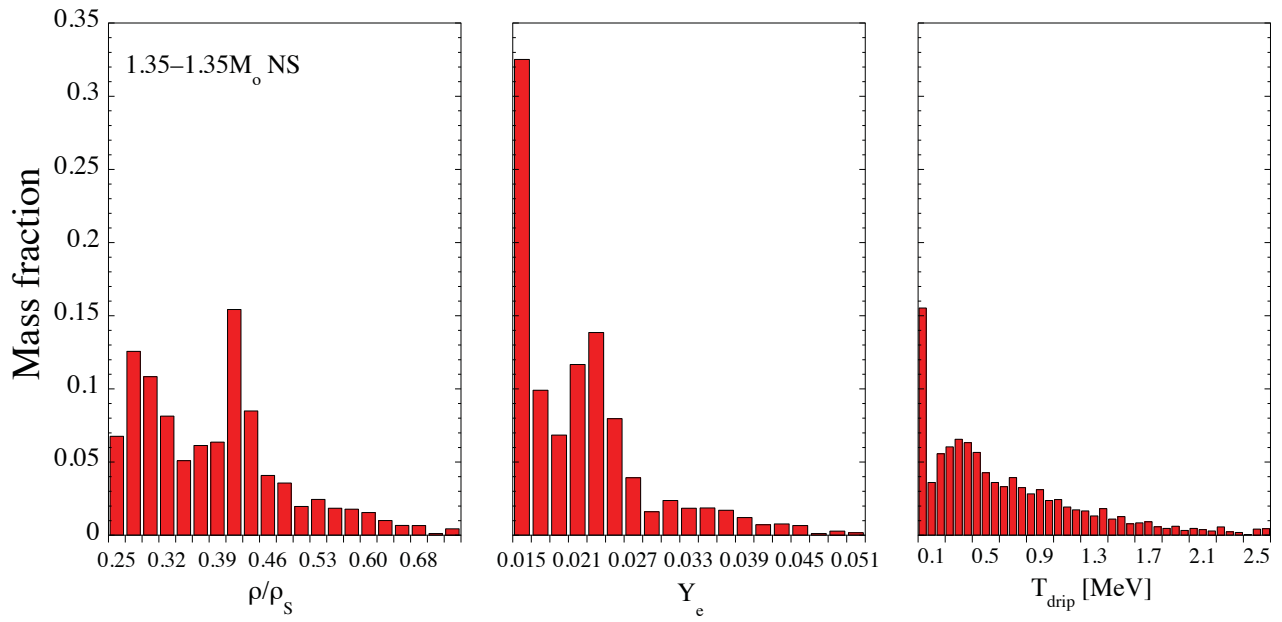

Fig. 13. Left: histogram representing the mass distribution of the ejected particles from a 1.35-1.35 $M_{\odot}$ NS binary system in different bins of initial density $\rho$ (relative to the saturation density $\rho_{S} \simeq 2.6 \times 10^{14} \mathrm{~g} / \mathrm{cm}^{3}$ ); Middle: same as the left panel for the electron fraction $Y_{e}$; Right: Same as the left panel for the temperature reached at the drip density 


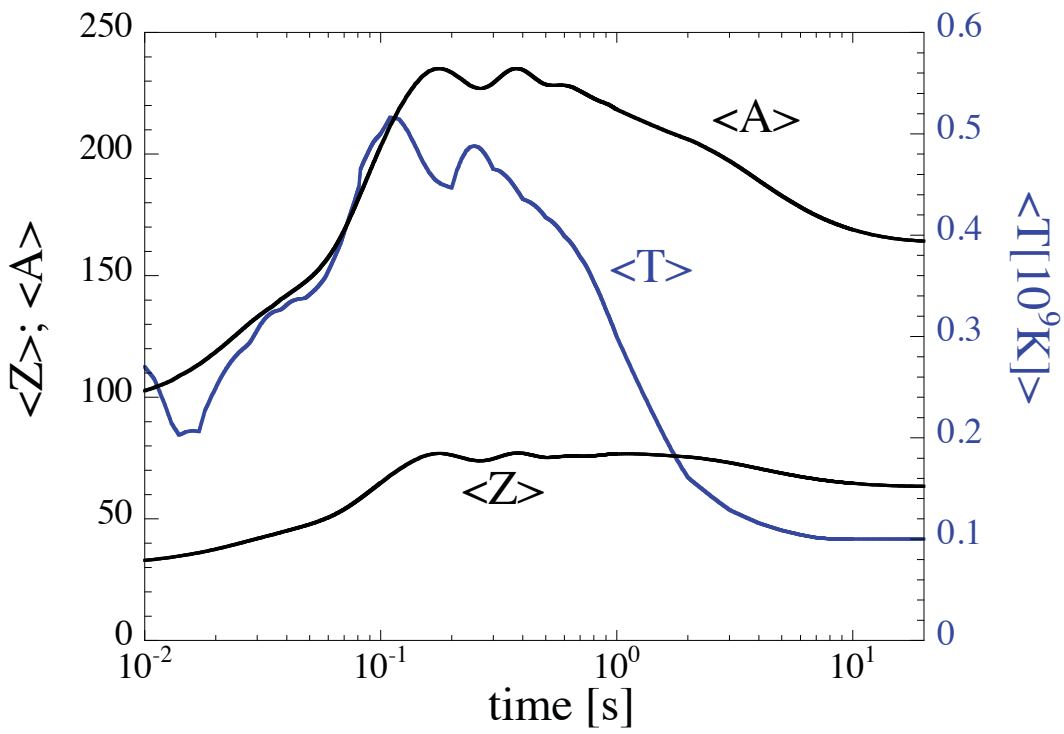

Fig. 14. Time variation of the charge number $\langle Z\rangle$, mass number $\langle A\rangle$ and temperature $\langle T\rangle$ mass-averaged over all the ejected particles

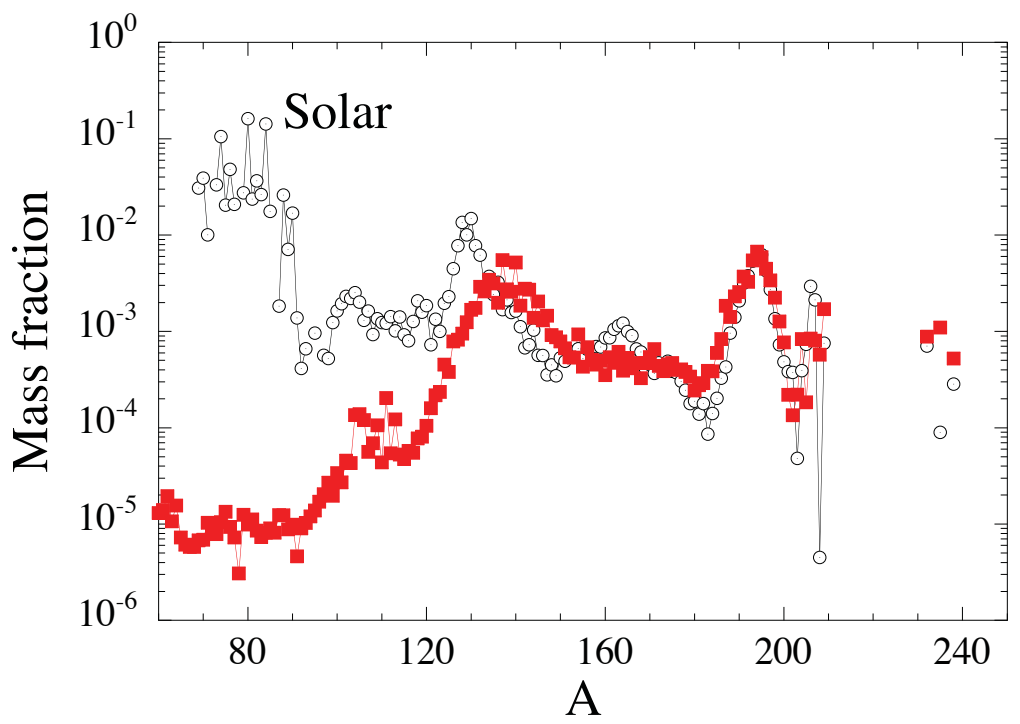

Fig. 15. Final abundance distribution of the matter ejected from the 1.35-1.35 $M_{\odot}$ NS merger as a function of the atomic mass. The distribution is compared with the solar $r$-abundance distribution (open dots) 
is found to be almost insensitive to all input parameters, such as the initial abundances, the expansion timescales or the adopted nuclear models. In contrast, the second peak around $A=140$ originates exclusively from the fission recycling, which is found to take place in the $A \simeq 280-290$ region at the time all the neutrons have been captured. These nuclei are found to fission symmetrically, as seen in Fig. 15 where the $A \simeq 140$ peak corresponds to the mass-symmetric fragment distribution. It should be emphasized that significant uncertainties still affect the prediction of fission probabilities and fragment distributions, so that the exact strength and location of the $A \simeq 140$ fission peak depend on the adopted nuclear model.

Although the outer crust $\left(\rho \leq \rho_{\text {drip }} \simeq 4.2 \times 10^{11} \mathrm{~g} / \mathrm{cm}^{3}\right.$ ) is far less massive than the inner crust, the ejection of the inner crust cannot take place without leading at the same time to the ejection of at least some outer crust material. The whole outer crust typically amounts to $10^{-5}$ to $10^{-4} M_{\odot}$, depending on the NS mass and radius (Pearson et al., 2011), so that its contribution, even for complete ejection, remains negligible compared to the $10^{-3}$ to $10^{-2} M_{\odot}$ of the inner crust material ejected during a NS-NS merger event. Still, the composition of the outer crust initially heated to NSE at a temperature of about $10^{10} \mathrm{~K}$ can be estimated before and after the decompression that follows its ejection (Goriely et al., 2011). Note that the amount of unbound outer crust and the dynamics of its ejection cannot be reliably calculated from the hydrodynamical model. Details about the adopted decompression model can be found in Goriely et al. (2011).

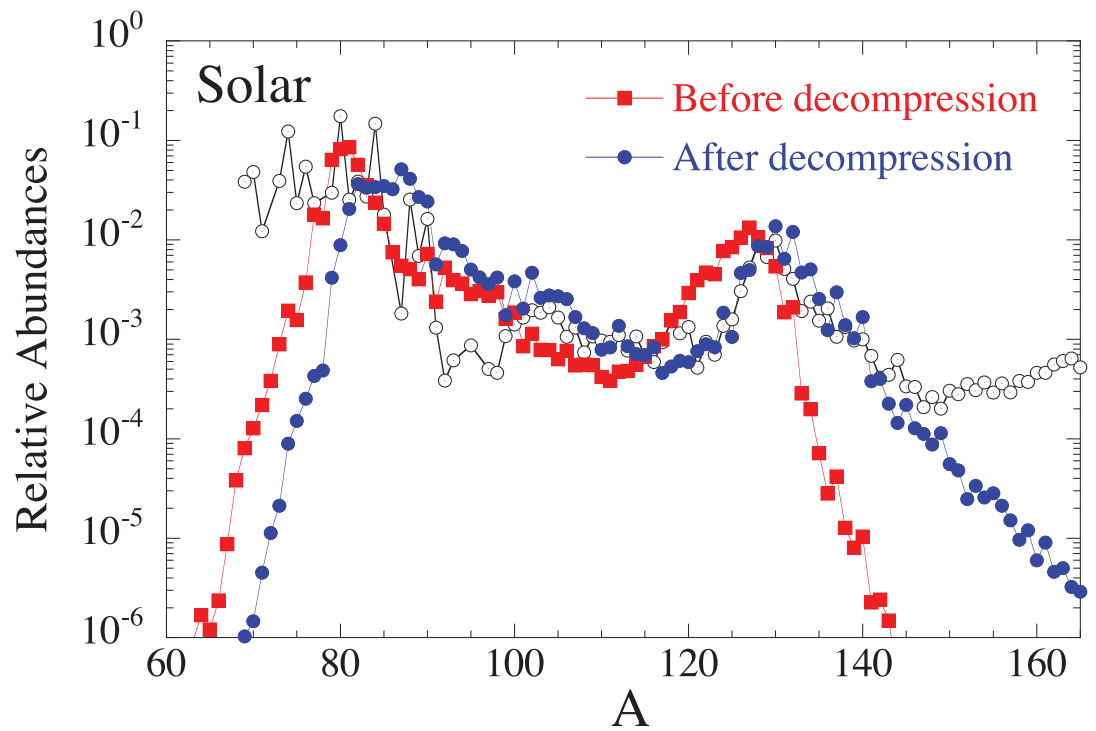

Fig. 16. Abundance distribution before and after decompression of a layer initially in NSE at $T_{9}=8$ and $\rho_{0}=3.4 \times 10^{11} \mathrm{~g} / \mathrm{cm}^{3}$ with initial pressure $P_{0}=4 \times 10^{-4} \mathrm{MeV} / \mathrm{fm}^{3}$. The distributions are compared with the solar r-abundance distributions (open dots). See Goriely et al. (2011) for details

Before decompression and at temperatures corresponding to $8-10 \times 10^{9} \mathrm{~K}$, the Coulomb effect due to the high densities in the crust leads to an overall content in neutron-rich nuclei of the outer crust close to the SoS r-abundance distribution, as shown in Fig. 16. Such a 
distribution differs, however, from the SoS one due to a shift of the second peak to lower mass numbers. During the decompression, the free neutrons (initially liberated as a result of the high temperatures) are re-captured, which leads to a final distribution of stable neutron-rich nuclei with a mass distribution of $80 \leq A \leq 140$ nuclei in excellent agreement with the SoS one, provided that the outer crust is initially at a temperature around $8 \times 10^{9} \mathrm{~K}$, and that all the layers of the outer crust are ejected. The decompression of the outer NS crust provides suitable conditions for a robust r-processing of the r-nuclides with $A \leq 140$. The overall abundance distribution depends on the fraction of the ejected crust, but also on the initial temperature at which the NSE has been frozen in. Figure 17 shows the abundance distributions obtained for different initial temperatures between 7 and $10 \times 10^{9} \mathrm{~K}$, assuming that the whole outer crust is ejected. The final composition should carry the imprint of the temperature at which the NSE is frozen prior to the ejection. Temperatures typically around $8-9 \times 10^{9} \mathrm{~K}$ correspond to those at which NSE can be dynamically achieved in cooling events Goriely et al. (2011).

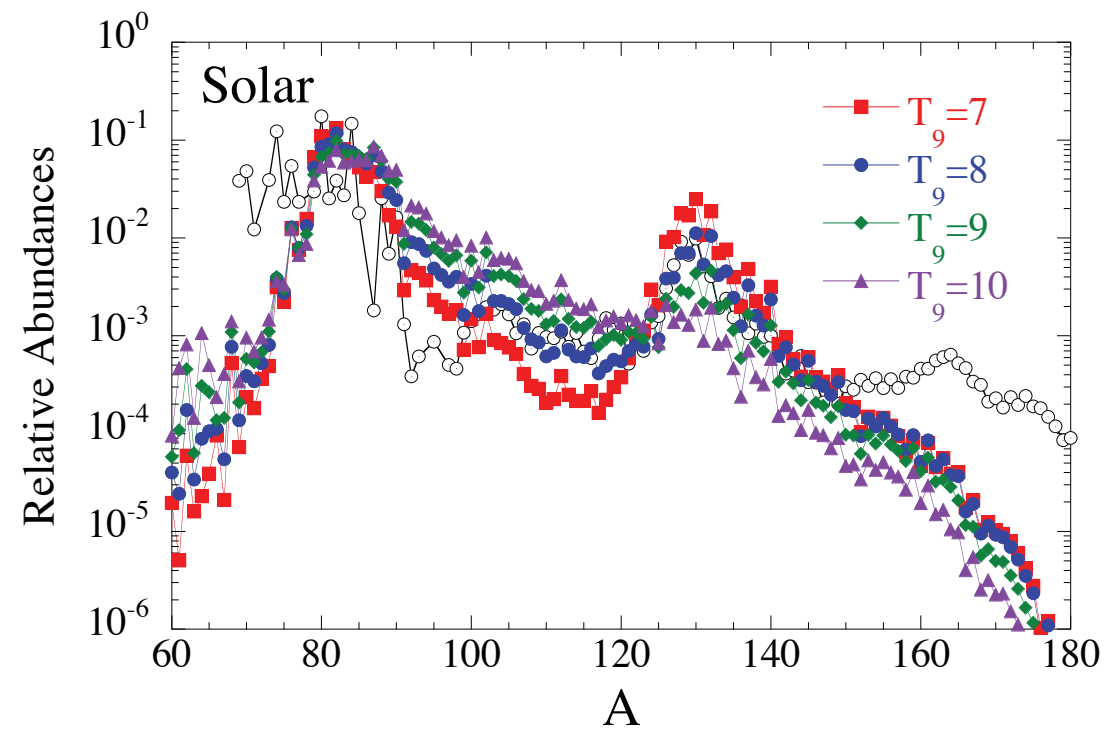

Fig. 17. Final abundance distributions of the outer crust material after decompression, if initially in NSE at different temperatures ranging between $T_{9}=7$ and 10 . The distributions are compared with the SoS r-abundance distributions (open dots). See Goriely et al. (2011) for details

It could be seen as purely fortuitous that temperatures around $T_{9} \simeq 8$ give rise to $r$-abundance distributions in agreement with the solar distribution. In fact, the temperatures considered here typically correspond to those at which NSE can be dynamically achieved in cooling events. As shown in Goriely et al. (2011), for matter with initial values of $Y_{e}=0.33-0.40$, it takes about 1 to $20 \mathrm{~ms}$ to reach a NSE at $T_{9}=8$ and $\rho=3 \times 10^{11} \mathrm{~g} / \mathrm{cm}^{-3}$, while at $T_{9} \simeq 9$, around $0.2 \mathrm{~ms}$ are required for the most neutron-rich conditions $\left(Y_{e}=0.33\right)$. Such ms timescales are characteristic of dynamical scenarios of interest here for the potential mass ejection (e.g. in the bursts of soft gamma-repeaters, during NS mergers, ...), so that prior to the ejection the NSE should be achieved at temperatures typically above $T_{9} \simeq 8-9$. It can be expected to be frozen during the ejection. 


\section{The evolution of the r-nuclide content of the Galaxy and nucleo-cosmochronology: inquietudes in a minefield}

A quite natural astrophysicists' dream is to understand the wealth of data on the evolution of the r-nuclide content of the Galaxy that are accumulating from very many spectroscopic observations (Sect. 4). These observations clearly demonstrate a huge complexity that will probably keep rising as new observations become available. The best one can thus imagine at this stage is to explain broad trends which may be identified through the analysis of the r-nuclide abundance information. In this exercise, one has always to keep in mind that, at best, more or less reliable elemental abundances are derived from spectral analyses that often rely on approximate classically used techniques. This necessitates to disentangle the s- and r-process contributions to a given elemental abundance. It is generally done by assuming that these two nucleosynthesis contributions are at a largely metallicity-independent relative level, and thus do not differ widely from the SoS case. This assumption cannot yet be ascertained in any quantitative way, and is in fact not expected to hold for the s-process.

Various galactic chemical evolution toy models have been constructed, which often focus on the evolution of the abundances of two representative elements, $\mathrm{Ba}$ (a s-process element in the SoS) and $\mathrm{Eu}$ (a r-process element in the SoS). They adopt different schematic descriptions of the galactic halo and disk, and different prescriptions for the physical input quantities to these models. In particular, fully ad-hoc assumptions are made or free parameters are chosen concerning the r-process yields from stars of different masses and metallicities.

Some predictions for [Eu/Fe] have been made recently by Wanajo \& Ishimaru (2006). They are based on a homogeneous one zone model in which it is assumed that stars in prescribed mass ranges produce an artificially selected amount of r-nuclides through the neutrino wind or prompt explosion mechanisms (Sect. 7.2). As expected, the predicted [Eu/Fe] ratio is very sensitive to the selected stellar mass ranges. This result might be optimistically considered as providing a way to constrain the site(s) of the r-process from observation. Reality is most likely less rosy, as very many uncertainties and severe approximations drastically blur the picture.

The assumption of the homogeneity of the interstellar medium at all times is dropped by Argast et al. (2004). The resulting inhomogeneous model might increase the plausibility of the predictions especially at early times in the galactic history. With the granularity of the nucleosynthesis events duly considered, one might hope to better account for the large observed scatter of the r-nuclide abundances at very low metallicities. In addition, the model of Argast et al. (2004) takes into account the r-process contribution from NS mergers (Sect. 8) on top of the one from $\mathrm{SNe}$ in selected mass ranges. The many other simplifications generally made in other chemical evolution models are also adopted by Argast et al. (2004). This concerns in particular the r-process yields from $\mathrm{SNe}$, as well as from NS mergers, that are just taken to be SoS-like. From their predicted [Eu/Fe] ratio, Argast et al. (2004) conclude that the scenario assuming the predominance of SNII events in the 20 to $50 M_{\odot}$ range allows the best fit to the observations. This result is obtained for total masses of r-nuclides per SN varying from about $10^{-4} M_{\odot}$ down to about $10^{-7} M_{\odot}$ when going from 20 to $50 M_{\odot}$ stars. Again, this conclusion has to be taken with great care in view of the many uncertainties and approximations involved in the chemical evolution model. Within the same model, it is also claimed that NS mergers are ruled out as the major source of r-nuclides in the Galaxy. This conclusion relies on a very approximate and highly uncertain time-dependent frequency of the events. In order to cope at best with observational constraints, coalescence timescales and 
amount of r-nuclides ejected per merger are adopted by Argast et al. (2004) to vary from about 0.1 to $10^{-4} M_{\odot}$, depending upon other parameters of the NS merging model.

All in all, we consider that the galactic chemical evolution models devised up to now are by far too schematic and uncertain to provide a reliable tool to account for the observed evolution of the r-nuclide content of the Galaxy, or for constraining the possible sites of the r-process. A priority should clearly be at least to identify with a reasonable confidence a single site for the r-process before dwelling on the grand project of constructing models for the evolution of the $r$-nuclide content of a whole galaxy, and of the SoS in particular.

As far as nucleo-cosmochronology is concerned, the actinides produced by the r-process enter in particular attempts to estimate the age of the Galaxy through their present SoS content, or through their abundances evaluated at the surface of very metal-poor stars. These attempts face some severe problems related to the nuclear physics and astrophysics uncertainties that affect the predictions of the actinide production. The situation is worsened further by the especially large uncertainties in the contribution of the r-process to the solar-system $\mathrm{Pb}$ and Bi content (Arnould et al., 2007). Concerning the ${ }^{232} \mathrm{Th}-{ }^{238} \mathrm{U}$ and ${ }^{235} \mathrm{U}-{ }^{238} \mathrm{U}$ pairs classically used to date the Galaxy from their present meteoritic abundances, the opinion has been expressed more than 20 years ago by Yokoi et al. (1983) that they have just limited chronometric virtues. This is in marked contrast to a widely-spread and repeated claim following Fowler \& Hoyle (1960), and based on simple analytic models for the evolution of Th and U in the Galaxy. In addition, the chronometric predictions based on the observations of Th and $U$ in very metal-poor stars have to be considered with great care (see Arnould et al., 2007, for a brief review and references). In order for them to be reliable, it is not only required that the production of the actinides by the r-process is well known, but also, and very decisively, that the production of Th with respect to $\mathrm{U}$ and to the Pt peak is universal. Observation demonstrates now that this is not the case. In spite of this, age evaluations based on this technique continue to appear in the literature.

\section{By way of a very brief conclusion}

The bottom line of this brief review of the r-process is that unanswered questions are by far more numerous than solved problems when one is dealing with this nucleosynthesis mechanism. They concern especially the astrophysics of the process, as no single site has been identified decisively yet. It also raises many nuclear physics questions. In such conditions, the modelling of the evolution of the r-nuclide content of the Galaxy and actinide-based chronometric evaluations cannot be put on solid grounds yet. This is in fact a very pleasing situation, as hope for many exciting discoveries is still ahead of us!

\section{References}

Argast, D., et al. (2004), Astron. Astrophys., 416, 997

Arlandini, C, et al. (1999). Astrophys. J., 525, 886

Arnould, M., \& Goriely, S. (2003). Phys. Rep., 384, 1

Arnould, M., Goriely, S., \& Takahashi, K.(2003), Phys. Rep., 384, 1

Banerjee, P., Haxton, W.C., \& Qian, Y.-Z. (2011), Phys. Rev. Lett., 106, 201104

Baym, G., Bethe, H.A., \& Pethick, C.J. (1971) Nucl. Phys. A, 175, 225

Clayton, D.D. (1968), Principles of Stellar Evolution and Nucleosynthesis (Chicago: Univ. Chicago Press)

Delano, M.D. \& Cameron, A.G.W. (1971), Astophys. Space Sci., 10, 203

Dillmann, I., \& Litvinov, Yu.A. (2011), Prog. Part. Nucl. Phys., 66, 358 
Duan, H., \& Friedland, A. (2011) Phys. Rev. Lett., 106, 091101

Duan, H., et al. (2011) J. Phys. G: Nucl. Part. Phys. 38, 035201

Epstein, R.I., Colgate, S.A., \& Haxton, W.C. (1988), Phys. Rev. Lett., 61, 2038

Fowler, W.A., \& Hoyle, F. (1960), Ann. Phys. 10, 280

Freiburghaus, C., Rosswog, S., \& Thielemann, F.-K. (1999), Astrophys. J., 525, L121

Gallagher, A.J., et al. (2010), Astron. Astrophys., 523, A24

Goriely, S. (1997), Astron. Astrophys., 327, 845

Goriely, S. (1999), Astron. Astrophys., 342, 881

Goriely, S., \& Siess, L. (2005) in From Lithium to Uranium: Elemental tracers of early cosmic evolution eds: V. Hill et al., Proc. of IAU symposium Nr 228, (Cambridge: Cambridge University Press), p. 451

Goriely, S., et al. (2011), Astron. Astrophys., 531, A78

Goriely, S., Bauswein, A., \& Janka, H.-T. (2011), Astrophys. J., 738, L32

Habs, D., et al. (2011), Appl. Phys. B 103, 471

Hoffman, R.D., et al. (1997), Astrophys. J., 482, 951

Janka, H.T., et al. (1999), Astrophys. J., 527, L39

Kitaura, F.S., et al. (2006), Astron. Astrophys., 450, 345

Lattimer, J.M., et al. (1977), Astrophys. J., 213, 225

Liebendörfer, M. et al. (2005), Astrophys. J., 620, 840

Lodders, K. (2010). In: Principles and Perspectives in Cosmochemistry, A. Goswami \& B.E. Reddy , (Ed.), 379-417, Springer-Verlag Berlin Heidelberg

Metzger, B.D., et al. (2010), Mon. Not. Roy. Astron. Soc., 406, 2650

Meyer, B.S. 1989, Astrophys. J., 343, 254

Meyer, B.S., et al. (1998), Astrophys. J., 498, 808

Oechslin, R., Janka, H.-T., \& Marek, A. (2007), Astron. Astrophys., 467, 395

Palme, H., \& Beer, H. (1993), in Landolt Börnstein, New Series, Group VI, Astron. \& Astrophys., Vol. 3, Subvol. a, (Berlin: Springer), p. 196

Pearson, J.M., Goriely, S., \& Chamel, N. (2011), Phys. Rev. C, 83, 065810

Roberts, L. F., Woosley, S. E.; \& Hoffman, R. D. (2010) Astrophys. J., 722, 954

Roberts, L.F., et al. 2011, Astrophys. J., 736, L21

Roederer, I.U. (2011) Astrophys. J. Let. 732, L17

Rosswog, S., Speith, R., \& Wynn, G.A. (2004), Mon. Not. Roy. Astron. Soc., 351, 1121

Qian, Y.-Z., \& Woosley, S.E. (1996), Astrophys. J., 471, 331

Simmerer, J., et al. (2004), Astrophys. J., 617, 1091

Takahashi, K., et al. (1994), Astron. Astrophys., 286, 857

Tsuruta, S., \& Cameron, A.G.W. (1965), Can. J. Phys. 43, 2056

Wanajo, S., \& Ishimaru, Y. (2006). Nucl. Phys. A77, 676c

Wanajo, S., et al. (2009), Astrophys. J., 695, 208

Wanajo, S., et al. (2011), Astrophys. J., 726, L15

Woosley, S.E., \& Hoffman, R.D. (1992), Astrophys. J., 395, 202

Woosley, S.E., et al. (1994), Astrophys. J., 433, 229

Yokoi, K., et al. (1983), Astron. Astrophys., 117, 65 


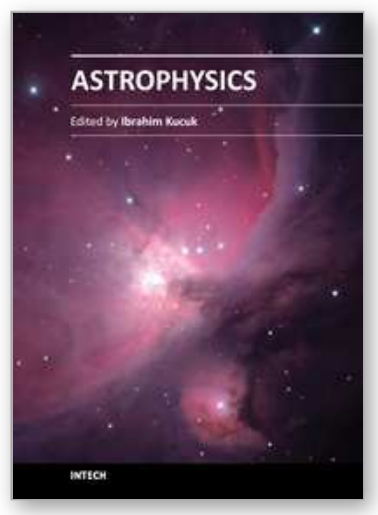

\author{
Astrophysics \\ Edited by Prof. Ibrahim Kucuk
}

ISBN 978-953-51-0473-5

Hard cover, 398 pages

Publisher InTech

Published online 30, March, 2012

Published in print edition March, 2012

This book provides readers with a clear progress to theoretical and observational astrophysics. It is not surprising that astrophysics is continually growing because very sophisticated telescopes are being developed and they bring the universe closer and make it accessible. Astrophysics Book presents a unique opportunity for readers to demonstrate processes do occur in Nature. The unique feature of this book is to cover different aspects in astrophysics covering the topics: • Astronomy • Theoretical Astrophysics • Observational Astrophysics • Cosmology • The Solar System • Stars • Planets • Galaxies • Observation • Spectroscopy • Dark Matter • Neutron Stars • High Energy Astrophysics

\title{
How to reference
}

In order to correctly reference this scholarly work, feel free to copy and paste the following:

Marcel Arnould and Stephane Goriely (2012). The r-Process of Nucleosynthesis: The Puzzle Is Still with Us, Astrophysics, Prof. Ibrahim Kucuk (Ed.), ISBN: 978-953-51-0473-5, InTech, Available from:

http://www.intechopen.com/books/astrophysics/the-r-process-of-nucleosynthesis-the-puzzle-is-still-with-us

\section{INTECH}

open science | open minds

\section{InTech Europe}

University Campus STeP Ri

Slavka Krautzeka 83/A

51000 Rijeka, Croatia

Phone: +385 (51) 770447

Fax: +385 (51) 686166

www.intechopen.com

\section{InTech China}

Unit 405, Office Block, Hotel Equatorial Shanghai

No.65, Yan An Road (West), Shanghai, 200040, China 中国上海市延安西路65号上海国际贵都大饭店办公楼 405 单元

Phone: +86-21-62489820

Fax: +86-21-62489821 
(C) 2012 The Author(s). Licensee IntechOpen. This is an open access article distributed under the terms of the Creative Commons Attribution 3.0 License, which permits unrestricted use, distribution, and reproduction in any medium, provided the original work is properly cited. 